\title{
Multicomponent fluids of hard hyperspheres in odd dimensions
}

\author{
René D. Rohrmann* \\ Instituto de Ciencias Astronómicas, de la Tierra y del Espacio (ICATE-CONICET), Avenida España 1512 Sur, 5400 San Juan, Argentina
}

\author{
Andrés Santos ${ }^{\dagger}$ \\ Departamento de Física, Universidad de Extremadura, E-06071 Badajoz, Spain
}

(Received 21 October 2010; published 21 January 2011)

\begin{abstract}
Mixtures of hard hyperspheres in odd-space dimensionalities are studied with an analytical approximation method. This technique is based on the so-called rational function approximation and provides a procedure for evaluating equations of state, structure factors, radial distribution functions, and direct correlation functions of additive mixtures of hard hyperspheres with any number of components and in arbitrary odd-dimension space. The method gives the exact solution of the Ornstein-Zernike equation coupled with the Percus-Yevick closure, thus, extending the solution for hard-sphere mixtures [J. L. Lebowitz, Phys. Rev. 133, A895 (1964)] to arbitrary odd dimensions. Explicit evaluations for binary mixtures in five dimensions are performed. The results are compared with computer simulations, and a good agreement is found.
\end{abstract}

DOI: 10.1103/PhysRevE.83.011201

PACS number(s): 61.20.Gy, 61.20.Ne, 05.20.Jj, 51.30.+i

\section{INTRODUCTION}

Systems made of hard bodies, i.e., impenetrable particles interacting solely through hard-core repulsions constitute useful simple fluid models [1]. Multicomponent hard-sphere mixtures serve as important reference systems in condensed matter and are relevant for understating the behavior of complex fluids with additional interparticle interactions, such as those in colloidal systems. These simple models capture the main features of the packing effects at short distances as they also occur in fluids governed by additional attractive interactions. Besides, the knowledge of the structural properties of hard-sphere fluids is a prerequisite for treating attractive interactions perturbatively and within a density-functional theory approach.

The study of $d$-dimensional hard-sphere fluids may prove to be a useful guide for investigating the solution of the threedimensional (3D) problem, apart from its own importance at a fundamental level. This explains the continued interest on hard-hypersphere systems found in the literature throughout the years [2-74]. However, multicomponent fluids at dimension $d>3$ have received much less attention, and the available information is rather sparse. The performed studies reduce to some evaluations of equations of state and virial coefficients $[18,30,34,44]$, phase-transition analyses [37], and computer simulations [40]. To our knowledge, none of the widely used mechanical-statistical theories (e.g., integral equation theories) have been applied to these systems.

The Percus-Yevick (PY) theory [75] is one of the classical approximations of liquid-state theory and, certainly, one of the most widely used. In the case of hard particles, exact PY solutions have been found for single-component fluids in odd dimensions, $d=1$ [76], $d=3$ [77,78], $d=5[3,7], d=7$ $[45,62], d=9,11$ [65], and also recently for even dimensions, $d=2$ [79] and $d=4,6,8$ [67]. Nevertheless, in the case of mixtures, PY solutions were provided only for the $3 \mathrm{D}$ fluid

\footnotetext{
*rohr@icate-conicet.gob.ar; http://icate-conicet.gob.ar/rohrmann †andres@unex.es; http://www.unex.es/eweb/fisteor/andres
}

[80,81], apart from the exact solution known for the mixture of hard rods [82]. The present paper is an attempt to cover the gap on hard particle mixtures at dimensions higher than three using the so-called rational function approximation (RFA).

The RFA approach was originally developed for hardsphere fluids [83]. The method was successfully applied to other related systems [84], such as hard-sphere mixtures [81], sticky hard spheres [85-87], square-well fluids [88,89], penetrable spheres [90], and one-component hyperspheres $[65,69]$. As we showed in Ref. [65], the RFA method, in its simplest version, recovers the exact PY solution for one-component hypersphere fluids in any space of odd dimension.

The aim of this paper is to extend the RFA theory to additive mixtures of hyperspheres in odd-dimensional Euclidean space. It is shown that the RFA method yields the exact solution of the Ornstein-Zernike (OZ) equation with the PY closure. While the method is generalized to any odd dimension, we focus, in particular, on the solution for the five-dimensional (5D) system. For this system, we analyze some of its thermodynamic and structural properties in the case of binary mixtures and compare them with available simulation data.

The remainder of this paper is organized as follows. Section II briefly describes some basic quantities of the equilibrium theory for multicomponent fluids of hard hyperspheres. In Sec. III, we introduce a Laplace functional associated with the fluid structure factors and derive its general properties. In Sec. IV, we present the extension of the RFA method to mixtures. Explicit formulation for the 3D and $5 \mathrm{D}$ cases are given in Sec. V, where compelling arguments about the equivalence to the PY solution are offered. Detailed evaluations for binary mixtures in $d=5$ are presented in Sec. VI. Section VII is devoted to the conclusions. The most technical aspects of the paper are relegated to the appendices.

\section{GENERAL BACKGROUND}

Here, it is useful to give some definitions that will be used in the following. Let $\rho$ be the total number density of an $\mathcal{N}$ component hypersphere mixture, let $\left\{x_{i}\right\}$ (with $i=1, \ldots, \mathcal{N}$ ) 
be the set of mole fractions, and let $\left\{\sigma_{i}\right\}$ be the set of diameters. The overall packing fraction is $\eta=\sum_{i=1}^{\mathcal{N}} \eta_{i}$, where

$$
\eta_{i}=v_{d} \rho x_{i} \sigma_{i}^{d}
$$

is the partial packing fraction due to species $i$. Here, $v_{d}$ is the volume of a $d$-dimensional sphere of unit diameter. For $d=$ odd,

$$
v_{d}=\frac{(\pi / 2)^{(d-1) / 2}}{d ! !} .
$$

The structure factor $S_{i j}(k)$ for the particle pair $(i, j)$ is given by

$$
S_{i j}(k)=x_{i} \delta_{i j}+\rho x_{i} x_{j} \widehat{h}_{i j}(k),
$$

where

$$
\widehat{h}_{i j}(k)=\int d \mathbf{r} h_{i j}(r) e^{-i \mathbf{k} \cdot \mathbf{r}}
$$

is the Fourier transform of the total pair correlation function $h_{i j}(r)$, related to the radial distribution function $(\mathrm{rdf}) g_{i j}(r)$ by

$$
h_{i j}(r)=g_{i j}(r)-1 .
$$

The compressibility factor $Z$ may be written as

$$
Z \equiv \frac{p}{\rho k_{B} T}=1+\frac{2^{d-1} \eta}{\mu_{d}} \sum_{i, j=1}^{\mathcal{N}} x_{i} x_{j} \sigma_{i j}^{d} g_{i j}\left(\sigma_{i j}^{+}\right),
$$

where $p$ is the pressure, $k_{B}$ is the Boltzmann constant, and $T$ is the temperature,

$$
\mu_{m} \equiv \sum_{\ell=1}^{\mathcal{N}} x_{\ell} \sigma_{\ell}^{m}
$$

denotes the moments of the diameter distribution, and $g_{i j}\left(\sigma_{i j}^{+}\right)$ is the contact value of the rdf, $\sigma_{i j}$ being the minimum possible distance between particles $i$ and $j$. For additive mixtures considered here, one has

$$
\sigma_{i j}=\frac{\sigma_{i}+\sigma_{j}}{2} .
$$

In Eq. (2.6), we have used $\eta=v_{d} \rho \mu_{d}$.

The isothermal susceptibility $\chi$ is given by

$$
\chi^{-1} \equiv \frac{1}{k_{B} T}\left(\frac{\partial p}{\partial \rho}\right)_{T,\left\{x_{i}\right\}}=1-\rho \sum_{i, j=1}^{\mathcal{N}} x_{i} x_{j} \widehat{c}_{i j}(0),
$$

where $\widehat{c}_{i j}(k)$ is the Fourier transform of the direct correlation function $c_{i j}(r)$, which is defined by the OZ equation,

$$
\widehat{h}_{i j}(k)=\widehat{c}_{i j}(k)+\rho \sum_{\ell=1}^{\mathcal{N}} x_{\ell} \widehat{h}_{i \ell}(k) \widehat{c}_{\ell j}(k) .
$$

In matrix form, the $\mathrm{OZ}$ relation can be rewritten as (see, e.g., Ref. [81])

$$
\mathrm{I}-\widetilde{\mathrm{C}}(k)=[\mathrm{I}+\tilde{\mathrm{h}}(k)]^{-1},
$$

where I is the $\mathcal{N} \times \mathcal{N}$ unit matrix and $\widetilde{\mathrm{c}}(k)$ and $\tilde{\mathrm{h}}(k)$ are $\mathcal{N} \times$ $\mathcal{N}$ matrices with elements $\rho \sqrt{x_{i} x_{j}} \widehat{c}_{i j}(k)$ and $\rho \sqrt{x_{i} x_{j}} \widehat{h}_{i j}(k)$, respectively. The compressibility equation of state (2.9) can be written as

$$
\begin{aligned}
\chi^{-1} & =\sum_{i, j=1}^{\mathcal{N}} \sqrt{x_{i} x_{j}}[\mathrm{I}-\tilde{\mathrm{c}}(0)]_{i j} \\
& =\sum_{i, j=1}^{\mathcal{N}} \sqrt{x_{i} x_{j}}[\mathrm{I}+\tilde{\mathrm{h}}(0)]_{i j}^{-1},
\end{aligned}
$$

where, in the last step, use has been made of Eq. (2.11). In particular, in the case of binary mixtures $(\mathcal{N}=2), \chi$ takes the form

$$
\chi=\frac{\left[1+\rho x_{1} \widehat{h}_{11}(0)\right]\left[1+\rho x_{2} \widehat{h}_{22}(0)\right]-\rho^{2} x_{1} x_{2} \widehat{h}_{12}^{2}(0)}{1+\rho x_{1} x_{2}\left[\widehat{h}_{11}(0)+\widehat{h}_{22}(0)-2 \widehat{h}_{12}(0)\right]} .
$$

The zero wave number value of $\widehat{h}_{i j}(k)$ can be expressed as

$$
\widehat{h}_{i j}(0)=d 2^{d} v_{d} H_{i j, d-1},
$$

where

$$
H_{i j, m}=\int_{0}^{\infty} d r h_{i j}(r) r^{m}
$$

is the $m$ th moment of $h_{i j}(r)$.

\section{THE LAPLACE FUNCTIONAL $G_{i j}(s)$}

\section{A. Definition}

In analogy to the case of one-component fluids [65], we introduce the Laplace functional of the rdf in a Euclidean space of odd dimension $d$ as

$$
G_{i j}(s)=\int_{0}^{\infty} \operatorname{drrg}_{i j}(r) \theta_{n}(s r) e^{-s r},
$$

which is defined in terms of the reverse Bessel polynomial $\theta_{n}(t)$ of degree $n=(d-3) / 2$ :

$$
\theta_{n}(t)=\sum_{\ell=0}^{n} \omega_{n, \ell} t^{\ell}, \quad \omega_{n, \ell}=\frac{(2 n-\ell) !}{2^{n-\ell}(n-\ell) ! \ell !} .
$$

More details of these polynomials and their properties can be found in Ref. [65]. Here, we recall that the Fourier transform of the total correlation functions can be expressed in terms of $G_{i j}(s)$ as (see Appendix A)

$$
\widehat{h}_{i j}(k)=v_{d}\left[\frac{G_{i j}(s)-G_{i j}(-s)}{s^{d-2}}\right]_{s=i k},
$$

where

$$
v_{d} \equiv(-2 \pi)^{(d-1) / 2} .
$$

The structure factors readily follow from Eq. (2.3). We note that the knowledge of $G_{i j}(s)$ allows us to obtain all the structural and thermodynamic properties of a multicomponent hard $d$-sphere fluid.

\section{B. $G_{i j}(s)$ at long and short wave numbers}

Being $g_{i j}\left(r<\sigma_{i j}\right)=0$ for hard-hypersphere fluids, from Eq. (3.1), one obtains at long $s$ [65],

$$
\lim _{s \rightarrow \infty} s^{(5-d) / 2} e^{\sigma_{i j} s} G_{i j}(s)=\sigma_{i j}^{(d-1) / 2} g_{i j}\left(\sigma_{i j}^{+}\right) .
$$


This equation determines the contact values of the radial distribution function, and we will use this to obtain the virial equation of the state of the fluid through Eq. (2.6).

On the other hand, from Eqs. (3.1) and (A6), $G_{i j}(s)$ may be written in terms of the total correlation function as

$$
G_{i j}(s)=\frac{(d-2) ! !}{s^{2}}+\int_{0}^{\infty} d r r h_{i j}(r) \theta_{n}(s r) e^{-s r} .
$$

The Taylor expansion of $e^{-s r}$ yields [65]

$$
G_{i j}(s)=\frac{(d-2) ! !}{s^{2}}+\sum_{m=0}^{\infty} \alpha_{n, m} H_{i j, m+1} s^{m},
$$

where the numerical coefficients $\alpha_{n, m}$ are given by

$$
\alpha_{n, m}=\sum_{\ell=0}^{\min (n, m)} \frac{(-1)^{m-\ell}}{(m-\ell) !} \omega_{n, \ell}
$$

The first $n$ coefficients $\alpha_{n, m}$ with $m=2 q+1=$ odd $(q=$ $0, \ldots, n-1)$ vanish [65]. Therefore,

$$
\begin{aligned}
G_{i j}(s)= & \frac{(d-2) ! !}{s^{2}}+\sum_{m=0}^{\infty} \alpha_{n, 2 m} H_{i j, 2 m+1} s^{2 m} \\
& +\sum_{m=n}^{\infty} \alpha_{n, 2 m+1} H_{i j, 2 m+2} s^{2 m+1} .
\end{aligned}
$$

As a consistency test, note that Eq. (2.14) is reobtained from Eqs. (3.3) and (3.9), making use of Eq. (2.2) and $\alpha_{n, 2 n+1}=(-1)^{n+1} /(2 n+1)$ !!. Therefore, the expansion of $G_{i j}(s)$ in powers of $s$ allows one to identify $H_{i j, d-1}$ from Eq. (3.9). This, in turn, gives the compressibility equation of state via Eqs. (2.12) and (2.14).

To summarize, the behaviors of $G_{i j}(s)$ at long and short $s$ are directly connected to the thermodynamic variables $Z$ and $\chi$, respectively.

\section{C. $G_{i j}(s)$ at low density}

The lowest terms in the density expansion of the rdf,

$$
g_{i j}(r)=g_{i j}^{(0)}(r)+\rho g_{i j}^{(1)}(r)+O\left(\rho^{2}\right),
$$

are

$$
\begin{gathered}
g_{i j}^{(0)}(r)=\Theta\left(r-\sigma_{i j}\right), \\
g_{i j}^{(1)}(r)=\Theta\left(r-\sigma_{i j}\right) \sum_{\ell} x_{\ell} \Omega_{\sigma_{i \ell}, \sigma_{j \ell}}(r),
\end{gathered}
$$

where $\Theta(x)$ is Heaviside's step function and $\Omega_{a, b}(r)$ is the intersection volume of two hyperspheres of radii $a$ and $b$ whose centers are separated by a distance $r \leqslant a+b$. In Laplace space, one has

$$
G_{i j}(s)=G_{i j}^{(0)}(s)+\rho G_{i j}^{(1)}(s)+O\left(\rho^{2}\right),
$$

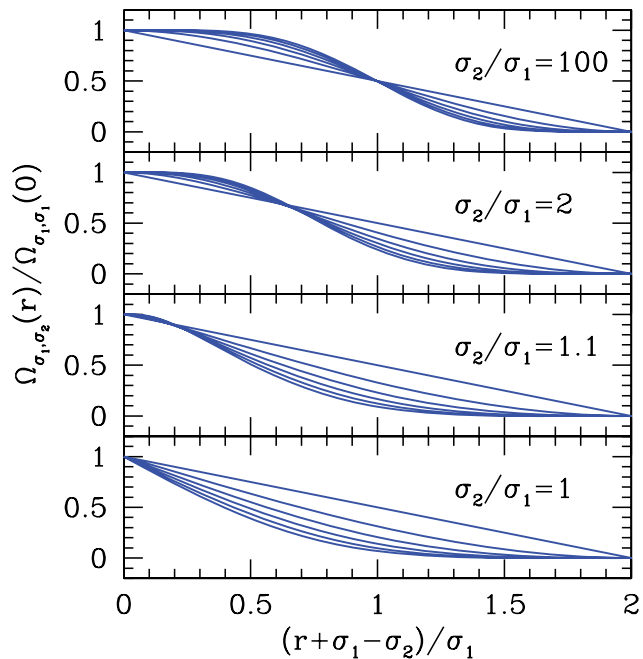

FIG. 1. (Color online) Scaled overlap volume $\Omega_{\sigma_{1}, \sigma_{2}}(r) / \Omega_{\sigma_{1}, \sigma_{1}}(0)$ of two hyperspheres with radii $\sigma_{1}$ and $\sigma_{2} \geqslant \sigma_{1}$ as a function of the center distance $r$, for Euclidean space with an odd dimension between 1 and 11 (lines starting from the straight line), and for several values of the size ratio $\sigma_{2} / \sigma_{1}$. The lower panel corresponds to two identical hyperspheres. The limit $\sigma_{2} \rightarrow \infty$ with $\sigma_{1}$ fixed represents a hypersphere of radius $\sigma_{1}$ crossing a flat wall.

with

$$
\begin{gathered}
G_{i j}^{(0)}(s)=\frac{\theta_{n+1}\left(\sigma_{i j} s\right) e^{-\sigma_{i j} s}}{s^{2}}, \\
G_{i j}^{(1)}(s)=\sum_{\ell=1}^{\mathcal{N}} x_{\ell} G_{i j \ell}^{(1)}(s), \\
G_{i j \ell}^{(1)}(s) \equiv \int_{\sigma_{i j}}^{\infty} d r r \theta_{n}(s r) \Omega_{\sigma_{i \ell}, \sigma_{j \ell}}(r) e^{-s r} .
\end{gathered}
$$

In Eq. (3.14), use has been made of Eq. (A7). To obtain $G_{i j \ell}^{(1)}(s)$, we first need the overlap volume $\Omega_{a, b}(r)$. An explicit expression for the latter quantity, valid for arbitrary radii and $d=$ odd, is derived in Appendix B [see Eqs. (B10) and (B16)]. Figure 1 shows graphs of the scaled intersection volume between two hyperspheres of radii $\sigma_{1}$ and $\sigma_{2} \geqslant \sigma_{1}$ as a function of the center separation $r$ (in rescaled units) for the first six odd dimensions $(d=1,3, \ldots, 11)$, and for several size ratios $\sigma_{2} / \sigma_{1}$

Inserting Eq. (B10) into the definition of $G_{i j \ell}^{(1)}(s)$, we get

$$
G_{i j \ell}^{(1)}(s)=(2 \pi)^{(d-1) / 2} \int_{\sigma_{i j}}^{\sigma_{i \ell}+\sigma_{j \ell}} d r \frac{R_{4 n+4}^{\left(\sigma_{i \ell}, \sigma_{j \ell}\right)}(r)}{r^{d-3}} \theta_{n}(s r) e^{-s r},
$$

where we have taken into account that $\sigma_{i j} \geqslant\left|\sigma_{i \ell}-\sigma_{j \ell}\right|=$ $\left|\sigma_{i}-\sigma_{j}\right| / 2$. It can be checked that $G_{i j \ell}^{(1)}(s)$ has the following structure:

$$
G_{i j \ell}^{(1)}(s)=\frac{v_{d}}{s^{d-2}}\left[G_{i \ell}^{(0)}(s) G_{j \ell}^{(0)}(s)+\frac{Q_{i j \ell}(s)}{s^{4}} e^{-\sigma_{i j} s}\right],
$$


where $Q_{i j \ell}(s)$ is a polynomial of degree $3 n+4=(3 d-1) / 2$, which can be further decomposed as

$$
\begin{aligned}
Q_{i j \ell}(s)= & s^{d+1} \bar{Q}_{i j \ell}(s)-\theta_{n+1}\left(\sigma_{i \ell} s\right) \sum_{m=0}^{n+1} \omega_{n+1, m}\left(\sigma_{j \ell} s\right)^{m} \\
& \times \sum_{q=0}^{2 n+3-m} \frac{\left(-\sigma_{\ell} s\right)^{q}}{q !},
\end{aligned}
$$

where $\bar{Q}_{i j \ell}(s)$ is a polynomial of degree $n=(d-3) / 2$. Note that $Q_{i j \ell}(s)=Q_{j i \ell}(s)$, but $\bar{Q}_{i j \ell}(s) \neq \bar{Q}_{j i \ell}(s)$.

It is worth pointing out that all the equations in this section and in Sec. II are exact.

\section{THE RFA}

In this section, we describe the RFA method to obtain the functional $G_{i j}(s)$, which, in turn, allows us to obtain the structural and thermodynamic properties of the hypersphere mixture. The method presented here is, on one hand, an extension to mixtures of the one recently applied to onecomponent systems of hyperspheres [65] and, on the other hand, an extension to hyperspheres of the one already proposed for mixtures of hard spheres $(d=3)$ [81].

The approximation we propose consists of assuming the following functional form:

$$
G_{i j}(s)=\frac{e^{-\sigma_{i j} s}}{s^{2}}\left[\mathrm{~L}(s) \cdot \mathrm{B}^{-1}(s)\right]_{i j},
$$

where $\mathrm{L}(s)$ and $\mathrm{B}(s)$ are $\mathcal{N} \times \mathcal{N}$ matrices given by

$$
\begin{gathered}
\mathrm{L}(s)=\sum_{m=0}^{n+1} \mathrm{~L}_{m} s^{m}, \\
\mathrm{~B}(s)=\mathrm{I}+\rho \sum_{m=0}^{n+1} \Phi_{m}(s) \cdot \mathrm{L}_{m} .
\end{gathered}
$$

Here, $\mathrm{L}_{m}, 0 \leqslant m \leqslant n+1$, are $\mathcal{N} \times \mathcal{N}$ matrices whose elements may depend on the fluid density, the particle diameters, and the mole fractions of the system, but are independent of $s$. Therefore, $\mathrm{L}(s)$ has a polynomial dependence on $s$ of degree $n+1=(d-1) / 2$. Besides, $\Phi_{m}(s)$ are diagonal matrices with elements given by

$$
\left[\Phi_{m}(s)\right]_{i i}=v_{d} x_{i} \sigma_{i}^{d-m-\delta} s^{-\delta} \phi_{d-m-\delta}\left(\sigma_{i} s\right),
$$

with

$$
\phi_{m}(x) \equiv \frac{1}{x^{m}}\left[\sum_{\ell=0}^{m} \frac{(-x)^{\ell}}{\ell !}-e^{-x}\right] .
$$

The series expansion of $\mathrm{B}(s)$ and its asymptotic long-s value are given in Appendix C. The parameter $\delta$ in Eq. (4.4) encompasses two different conditions of normalization for $\mathrm{B}(s)$, specifically [see Eqs. (C8) and (C11)],

$$
\lim _{s \rightarrow 0} \mathrm{~B}(s)=\mathrm{I} \quad(\delta=0), \quad \lim _{s \rightarrow \infty} \mathrm{B}(s)=\mathrm{I} \quad(\delta=1) .
$$

These cases constitute two alternative choices of the analytical representation for $G_{i j}(s)$ which, however, yield identical physical results (each one with its particular quantities $L_{m}$ ).
In the functional form (4.1) for $G_{i j}(s)$, we are using the constraints derived in Secs. III B and III C. In particular, as shown in Appendix D, Eq. (4.1) is consistent with the exact low-density expansion given by Eqs. (3.13), (3.14), (3.15), (3.18), and (3.19).

The number $n+2$ of terms in the representations of $\mathrm{L}(s)$ and $\mathrm{B}(s)$, Eqs. (4.2) and (4.3), is the minimum one required to verify the correct behavior of $G_{i j}(s)$ at large $s$. In fact, Eqs. (3.5) and (4.1) yield

$$
\begin{aligned}
\sigma_{i j}^{n+1} g_{i j}\left(\sigma_{i j}^{+}\right) & =\lim _{s \rightarrow \infty} s^{-(n+1)}\left[\mathrm{L}(s) \cdot \mathrm{B}^{-1}(s)\right]_{i j} \\
& =\left[\mathrm{L}_{n+1} \cdot \mathrm{B}^{-1}(\infty)\right]_{i j},
\end{aligned}
$$

with $\mathrm{B}(\infty)$ given by Eq. (C11). With the normalization choice $\delta=1$, the contact values $g_{i j}\left(\sigma_{i j}^{+}\right)$are directly related to the components of $L_{n+1}$,

$$
\sigma_{i j}^{(n+1)} g_{i j}\left(\sigma_{i j}^{+}\right)=\left(\mathrm{L}_{n+1}\right)_{i j} \quad(\delta=1) .
$$

Now, we want to determine the $n+1$ coefficients $L_{m}$. This is done by requiring consistency with Eq. (3.9). First, let us rewrite Eq. (3.7) as

$$
\frac{s^{2}}{(2 n+1) ! !} G_{i j}(s)=1+\sum_{m=0}^{\infty}\left(\Gamma_{m}\right)_{i j} s^{m-2},
$$

where we have introduced the matrices $\Gamma_{m}$ as

$$
\left(\Gamma_{m}\right)_{i j} \equiv \frac{\alpha_{n, m} H_{i j, m+1}}{(2 n+1) ! !} .
$$

Next, we note that

$$
e^{\sigma_{i j} s}=\sum_{m=0}^{\infty}\left(\Lambda_{m}\right)_{i j} s^{m},
$$

where

$$
\left(\wedge_{m}\right)_{i j} \equiv \frac{\sigma_{i j}^{m}}{m !}
$$

Consequently,

$$
\frac{s^{2}}{(2 n+1) ! !} e^{\sigma_{i j} s} G_{i j}(s)=\sum_{m=0}^{\infty}\left(\mathrm{K}_{m}\right)_{i j} s^{m},
$$

with

$$
\mathrm{K}_{m} \equiv \Lambda_{m}+\sum_{\ell=0}^{m-2} \Gamma_{\ell} \otimes \Lambda_{m-\ell-2}
$$

where the symbol $\otimes$ denotes a matrix product element to element: $(A \otimes B)_{i j} \equiv A_{i j} B_{i j}$.

Apart from the introduced notation, Eq. (4.13) is totally equivalent to Eq. (3.7). Now, according to the RFA form (4.1),

$$
\sum_{m=0}^{n+1} \tilde{\mathrm{L}}_{m} s^{m}=\left(\sum_{m=0}^{\infty} \mathrm{K}_{m} s^{m}\right) \cdot\left(\sum_{k=0}^{\infty} \mathrm{B}_{k} s^{k}\right),
$$

where

$$
\tilde{\mathrm{L}}_{m} \equiv \frac{\mathrm{L}_{m}}{(2 n+1) ! !}
$$


and the matrices $\mathrm{B}_{k}$ are given by Eqs. (C5) and (C6). From a power analysis of Eq. (4.15), one obtains

$$
\sum_{k=0}^{\ell} \mathrm{K}_{\ell-k} \cdot \mathrm{B}_{k}=\tilde{\mathrm{L}}_{\ell}
$$

with the convention $\widetilde{\mathrm{L}}_{\ell}=0$ if $\ell>n+1$. If we choose $\delta=0$, the first relation in Eq. (4.17) (i.e., $\ell=0$ ) is trivially solved and yields

$$
\left(\mathrm{L}_{0}\right)_{i j}=(2 n+1) ! ! \quad(\delta=0) .
$$

Since, as mentioned following Eq. (3.8), the first $n$ coefficient $\alpha_{n, m}$ with $m=$ odd $\leqslant 2 n-1$ vanish, we have

$$
\Gamma_{1}=\Gamma_{3}=\cdots=\Gamma_{2 n-1}=0 .
$$

The property (4.19) can be used, together with Eq. (4.17) with $\ell=$ even $\leqslant 2 n+2$, to express the matrices $\Gamma_{2 m}, 0 \leqslant m \leqslant n$, in terms of the matrices $L_{\ell}$ by means of the recursion relation

$$
\Gamma_{2 m}=\widetilde{\mathrm{L}}_{2 m+2}-\sum_{k=1}^{m+1} \sum_{\ell=0}^{2 k}\left[\Gamma_{2(m-k)} \otimes \Lambda_{2 k-\ell}\right] \cdot \mathrm{B}_{l},
$$

where we must adopt the convention $\left(\Gamma_{-2}\right)_{i j} \equiv 1$. Similarly, from Eq. (4.17) with $\ell=$ odd $\leqslant 2 n+1$, one obtains

$$
\tilde{\mathrm{L}}_{2 m+1}=\sum_{k=1}^{m+1} \sum_{\ell=0}^{2 k-1}\left[\Gamma_{2(m-k)} \otimes \Lambda_{2 k-\ell-1}\right] \cdot \mathrm{B}_{\ell},
$$

with $0 \leqslant m \leqslant n$. Once the matrices $\Gamma_{2 m}$ are obtained in terms of $L_{\ell}$ via Eq. (4.20), Eq. (4.21) becomes a closed system of $n+$ $1=(d-1) / 2$ matricial algebraic equations for the unknowns $\mathrm{L}_{1}, \mathrm{~L}_{2}, \ldots, \mathrm{L}_{n+1}$. Therefore, the problem of finding $G_{i j}(s)$ as given by Eq. (4.1) is reduced to solving Eqs. (4.20) and (4.21). In the case of hard-sphere mixtures $(d=3)$, it is possible to find an analytical solution, as proved in Ref. [81].

The solutions of Eq. (4.21) that are physically meaningful are those that verify the correct behavior in the limit $\rho \rightarrow 0$. For $\delta=0$, we find from Eqs. (3.13), (3.14), and (4.1),

$$
\lim _{\eta \rightarrow 0}\left(\mathrm{~L}_{m}\right)_{i j}=\omega_{n+1, m} \sigma_{i j}^{m},
$$

where the coefficients $\omega_{n+1, m}$ of the reverse Bessel polynomial $\theta_{n+1}(t)$ are given by Eq. (3.2). Alternatively, when all particle diameters adopt the same value (say $\sigma_{i j}=1 \forall i, j$ ), we must recover the pure-fluid solution, which only depends on the density or packing fraction (but not on the mole fractions), i.e.,

$$
\left.\left(\mathrm{L}_{m}\right)_{i j}\right|_{\sigma_{i j}=1}=a_{m}(\eta),
$$

where the functions $a_{m}(\eta)$ are defined in Ref. [65]. Appendix E shows that the one-component solution [65] is actually recovered from the approach (4.1).

Figure 2 illustrates the elements of the matrix $L_{2}$, as obtained from the physical solution of Eq. (4.21), for a 5D binary mixture $(d=5, n=1)$ as functions of the diameter $\sigma_{2}$ (with $\sigma_{1}=1$ ) for $x_{2}=\frac{1}{4}$ and several density values. The dashed lines correspond to the limit of zero density, as given by Eq. (4.22), while the symbols show the solution for a one-component fluid [see Eq. (4.23)], with $a_{2}(\eta)$ given by Eq. (E10) of Ref. [65].

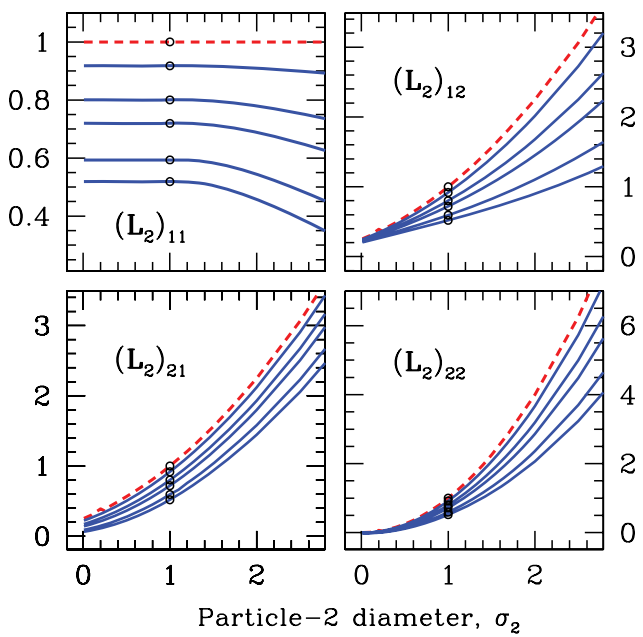

FIG. 2. (Color online) Matrix element of $\mathrm{L}_{2}$ for a binary mixture at $d=5$ as functions of the diameter $\sigma_{2}\left(\sigma_{1}=1\right)$ for $x_{2}=\frac{1}{4}$ and different values of the packing fraction. From top to bottom, the curves correspond to $\eta=0,0.01,0.02,0.05,0.1$, and 0.15 . The pure-fluid solutions are indicated by symbols.

Once the solutions of Eq. (4.21) are obtained, they may be used in Eq. (4.7) to obtain the contact values of the pair radial distribution functions and, subsequently, the compressibility factor in the so-called virial route using the equation of state (2.6). Its expression reads

$$
Z_{v}=1+\frac{2^{d-1} \eta}{\mu_{d}} \sum_{i, j=1}^{\mathcal{N}} x_{i} x_{j} \sigma_{i j}^{(d+1) / 2}\left[\mathrm{~L}_{n+1} \cdot \mathrm{B}^{-1}(\infty)\right]_{i j}
$$

Furthermore, the isothermal susceptibility $\chi$ of the fluid may be evaluated by means of the thermodynamical formula (2.9), using the relation resulting from Eq. (2.14) and the definition of $\Gamma_{m}$ in Eq. (4.10),

$$
\widehat{\mathrm{h}}(0)=2(-2 \pi)^{(d-1) / 2}(d-2) ! ! \Gamma_{2 n+1},
$$

with $\Gamma_{2 n+1}$ given by

$$
\Gamma_{2 n+1}=-\sum_{k=1}^{n+2} \sum_{\ell=0}^{2 k-1}\left[\Gamma_{2(n-k+1)} \otimes \Lambda_{2 k-\ell-1}\right] \cdot \mathrm{B}_{l},
$$

which proceeds from Eq. (4.17) at $\ell=2 n+3$, taking into account that $\widetilde{L}_{2 n+3}=0$. The values of the isothermal susceptibility can then be used to obtain the compressibility factor in the so-called compressibility route as

$$
Z_{c}(\eta)=\int_{0}^{1} d x \chi^{-1}(\eta x) .
$$

Of course, the structure factors and total correlation functions can be obtained easily with Eqs. (2.3) and (3.3), once the solutions $L_{m}$ have been substituted into Eq. (4.1). Notice that, as shown in Appendix D, Eq. (4.1) is exact to first order in $\rho$ so that the first three terms of an expansion of the right-hand side of Eq. (2.3), in powers of $\rho$, are exact within the RFA method. 


\section{EXPLICIT EXPRESSIONS}

In this section, we briefly revise the RFA solution for mixtures of hard spheres $(d=3, n=0)$ and derive results for 5D hyperspheres $(d=5, n=1)$. The $3 \mathrm{D}$ analysis from the present framework is useful as a guide for subsequent applications of the RFA method to mixtures in higher dimensions. Here, we use the version of the RFA approach based on the choice $\delta=0$.

For simplicity, henceforth, we introduce the following matrix notation:

$$
\begin{gathered}
\llbracket A_{i} \rrbracket_{\alpha \beta} \equiv A_{\alpha}, \quad \llbracket A_{j} \rrbracket_{\alpha \beta} \equiv A_{\beta}, \\
\llbracket A_{k} \rrbracket_{\alpha \beta} \equiv A_{\alpha} \delta_{\alpha \beta},
\end{gathered}
$$

This means that, given a list $\mathcal{A}=\left\{A_{1}, A_{2}, \ldots, A_{\mathcal{N}}\right\}$, $A_{i} \rrbracket$ represents the $\mathcal{N} \times \mathcal{N}$ square matrix made by repeating the list $\mathcal{A}$ as columns so that all the elements of a given row are equal. Analogously, $\llbracket A_{j} \rrbracket=\llbracket A_{i} \rrbracket^{\dagger}$ (where $\dagger$ indicates the transpose of a matrix) is obtained by repeating the list $\mathcal{A}$ as rows so that all the elements of a given column are equal. If the elements of $\mathcal{A}$ are placed along the main diagonal, one gets the diagonal matrix $\llbracket A_{k} \rrbracket$. The meaning of $\llbracket A_{i j} \rrbracket$ is self-evident. We will also use the notation [1] to refer to a matrix with all the elements equal to 1 . Note the properties $\llbracket C_{k} \rrbracket \cdot \llbracket A_{j} \rrbracket=$ $\llbracket C_{i} A_{j} \rrbracket, \llbracket C_{k} \rrbracket \cdot \llbracket A_{i} \rrbracket=\llbracket C_{i} A_{i} \rrbracket$, and $\llbracket A_{j} \rrbracket \cdot \llbracket C_{k} \rrbracket=\llbracket C_{j} A_{j} \rrbracket$, $\llbracket A_{i} \rrbracket \cdot \llbracket C_{k} \rrbracket=\llbracket A_{i} C_{j} \rrbracket$. Some inversion properties involving these matrices are proved in Appendix F.

\section{A. Hard-sphere mixtures}

For $d=3(n=0)$, Eq. (4.1) becomes

$$
G_{i j}(s)=\frac{e^{-\sigma_{i j} s}}{s^{2}}\left[\left(\mathrm{~L}_{0}+\mathrm{L}_{1} s\right) \cdot \mathrm{B}^{-1}(s)\right]_{i j},
$$

with

$$
\mathrm{B}(s)=\mathrm{I}+\rho\left[\Phi_{0}(s) \cdot \mathrm{L}_{0}+\Phi_{1}(s) \cdot \mathrm{L}_{1}\right],
$$

where $L_{0}$ and $L_{1}$ are the unknowns. From Eq. (4.18), we have $\mathrm{L}_{0}=\llbracket 1 \rrbracket$, and Eq. (4.21) yields

$$
\left(\mathrm{I}+\llbracket 2 \eta_{j} \rrbracket\right) \cdot \mathrm{L}_{1}=\llbracket \sigma_{i j} \rrbracket+\frac{\eta \mu_{4}}{2 \mu_{3}} \llbracket 1 \rrbracket,
$$

with $\mu_{m}$ defined by Eq. (2.7). Using Eq. (F1), it is straightforward to obtain

$$
\mathrm{L}_{1}=\frac{\llbracket \sigma_{i} \rrbracket}{2}+\frac{\llbracket \sigma_{j} \rrbracket}{2(1+2 \eta)}-\frac{\eta \mu_{4}}{2 \mu_{3}(1+2 \eta)} \llbracket 1 \rrbracket .
$$

This closes the determination of $G_{i j}(s)$.

In order to evaluate the contact values of $g_{i j}$ [see Eq. (4.7)],

$$
\sigma_{i j} g_{i j}\left(\sigma_{i j}^{+}\right)=\left[\mathrm{L}_{1} \cdot \mathrm{B}^{-1}(\infty)\right]_{i j},
$$

we must determine $\mathrm{B}(\infty)$. From Eq. (C11) (with $\delta=0$ ),

$$
\mathrm{B}(\infty)=\mathrm{I}+\llbracket \Delta_{i} \rrbracket+\llbracket \Omega_{i} \sigma_{j} \rrbracket,
$$

with

$$
\begin{gathered}
\Delta_{i} \equiv \frac{3 \eta}{1+2 \eta} \frac{\mu_{4}}{\mu_{3}} \frac{\eta_{i}}{\sigma_{i}}-\eta_{i}, \\
\Omega_{i} \equiv-\frac{3}{1+2 \eta} \frac{\eta_{i}}{\sigma_{i}} .
\end{gathered}
$$

Using the relation $(\mathrm{F} 4)$, it results

$$
\begin{aligned}
\mathrm{B}^{-1}(\infty)= & \mathrm{I}+\frac{1}{\gamma}\left\{\alpha \llbracket \Omega_{i} \rrbracket-(1+a) \llbracket \Omega_{i} \sigma_{j} \rrbracket\right. \\
& \left.-(1+\beta) \llbracket \Delta_{i} \rrbracket+b \llbracket \Delta_{i} \sigma_{j} \rrbracket\right\},
\end{aligned}
$$

where

$$
\begin{gathered}
a \equiv \operatorname{tr}\left(\llbracket \Delta_{i} \rrbracket\right)=-\eta\left(1+\beta \frac{\mu_{4} \mu_{2}}{\mu_{3}^{2}}\right), \\
b \equiv \operatorname{tr}\left(\llbracket \Omega_{i} \rrbracket\right)=\beta \frac{\mu_{2}}{\mu_{3}}, \\
\alpha \equiv \operatorname{tr}\left(\llbracket \Delta_{i} \sigma_{j} \rrbracket\right)=-\frac{\eta \mu_{4}}{\mu_{3}}(1+\beta), \\
\beta \equiv \operatorname{tr}\left(\llbracket \Omega_{i} \sigma_{j} \rrbracket\right)=-\frac{3 \eta}{1+2 \eta}, \\
\gamma \equiv(1+a)(1+\beta)-\alpha b=\frac{(1-\eta)^{2}}{1+2 \eta} .
\end{gathered}
$$

The matrix products on the right-hand side of Eq. (5.6) are

$$
\begin{aligned}
\llbracket \sigma_{i} \rrbracket \cdot \llbracket \Delta_{i} \rrbracket & =a \llbracket \sigma_{i} \rrbracket, \quad \llbracket \sigma_{j} \rrbracket \cdot \llbracket \Delta_{i} \rrbracket=\alpha \llbracket 1 \rrbracket, \\
\llbracket \sigma_{i} \rrbracket \cdot \llbracket \Delta_{i} \sigma_{j} \rrbracket & =a \llbracket \sigma_{i} \sigma_{j} \rrbracket, \quad \llbracket \sigma_{j} \rrbracket \cdot \llbracket \Delta_{i} \sigma_{j} \rrbracket=\alpha \llbracket \sigma_{j} \rrbracket, \\
\llbracket 1 \rrbracket \cdot \llbracket \Delta_{i} \rrbracket & =a \llbracket 1 \rrbracket, \quad \llbracket 1 \rrbracket \cdot \llbracket \Delta_{i} \sigma_{j} \rrbracket=a \llbracket \sigma_{j} \rrbracket,
\end{aligned}
$$

plus similar results in the case of $\llbracket \Omega_{i} \rrbracket$. Taking into account the preceding results, one obtains from Eq. (5.6),

$$
g_{i j}\left(\sigma_{i j}^{+}\right)=\frac{1}{1-\eta}+\frac{3 \eta}{2(1-\eta)^{2}} \frac{\mu_{2} \sigma_{i} \sigma_{j}}{\mu_{3} \sigma_{i j}} .
$$

Its application in Eq. (2.6) gives the pressure equation in the virial route obtained by Lebowitz [80].

On the other hand, thanks to Eq. (5.5), it is possible to solve Eqs. (4.25) and (4.26) analytically. The result is

$$
\begin{aligned}
\frac{\widehat{h}(0)}{\pi}= & \frac{\eta}{8}\left[\frac{9 \eta^{2}\left(\mu_{4} / \mu_{3}\right)^{3}}{(1+2 \eta)^{2}}-\frac{6 \eta \mu_{4} \mu_{5} / \mu_{3}^{2}}{1+2 \eta}+\frac{\mu_{6}}{\mu_{3}}\right] \llbracket 1 \rrbracket \\
& +\eta \frac{(1+2 \eta) \mu_{5} / \mu_{3}-3 \eta\left(\mu_{4} / \mu_{3}\right)^{2}}{(1+2 \eta)^{2}} \llbracket \sigma_{i j} \rrbracket \\
& -\frac{1}{8} \llbracket \sigma_{i}^{3}+\sigma_{j}^{3} \rrbracket \\
& +\frac{\eta\left(\mu_{4} / \mu_{3}\right)\left(\llbracket \sigma_{i j}^{2} \rrbracket+\llbracket \sigma_{i} \sigma_{j} \rrbracket\right)-\llbracket \sigma_{i}^{2} \sigma_{j}+\sigma_{i} \sigma_{j}^{2} \rrbracket}{2(1+2 \eta)}
\end{aligned}
$$

The use of Eq. (5.18) into Eq. (2.13) yields the PY solution for the isothermal susceptibility $\chi$, which agrees with that given by Ashcroft and Langreth [91] for a binary mixture of hard spheres.

\section{B. 5D mixtures}

For a fluid of hyperspheres in $d=5(n=1)$, the functional $G_{i j}(s)$ takes the form

$$
G_{i j}(s)=\frac{e^{-\sigma_{i j} s}}{s^{2}}\left[\left(\mathrm{~L}_{0}+\mathrm{L}_{1} s+\mathrm{L}_{2} s^{2}\right) \cdot \mathrm{B}^{-1}(s)\right]_{i j},
$$

with

$$
\mathrm{B}(s)=\mathrm{I}+\rho\left[\Phi_{0}(s) \cdot \mathrm{L}_{0}+\Phi_{1}(s) \cdot \mathrm{L}_{1}+\Phi_{2}(s) \cdot \mathrm{L}_{2}\right] .
$$


According to Eq. (4.18), $\mathrm{L}_{0}=3 \llbracket 1 \rrbracket$. Besides, Eq. (4.21) at $m=0$ yields [92]

$$
\begin{aligned}
\mathrm{L}_{1}= & 3 \llbracket \sigma_{i j} \rrbracket+\frac{3}{1-6 \eta} \\
& \times\left(2 \eta \frac{\mu_{6}}{\mu_{5}} \llbracket 1 \rrbracket+3 \eta \llbracket \sigma_{j} \rrbracket-10 \llbracket \eta_{j} / \sigma_{j} \rrbracket \cdot \mathrm{L}_{2}\right) .
\end{aligned}
$$

This expresses $L_{1}$ in terms of $L_{2}$. From Eq. (4.20), with $m=0$, we find

$$
\begin{aligned}
\Gamma_{0}= & -\eta\left[\frac{9 \mu_{7} / \mu_{5}}{14}+\frac{4 \eta\left(\mu_{6} / \mu_{5}\right)^{2}}{1-6 \eta}\right] \llbracket 1 \rrbracket-\frac{2 \eta\left(\mu_{6} / \mu_{5}\right) \llbracket \sigma_{i j} \rrbracket}{1-6 \eta} \\
& -\frac{3 \eta \llbracket \sigma_{i} \sigma_{j} \rrbracket}{2(1-6 \eta)}-\frac{1}{2} \llbracket \sigma_{i j} \rrbracket^{2}+\mathrm{Q}_{2} \cdot \mathrm{L}_{2},
\end{aligned}
$$

where

$$
\mathrm{Q}_{2} \equiv \frac{\mathrm{I}}{3}+3 \llbracket \eta_{j} \rrbracket+\frac{20 \eta \mu_{6} \llbracket \eta_{j} / \sigma_{j} \rrbracket}{\mu_{5}(1-6 \eta)}+\frac{5 \llbracket \eta_{j} \sigma_{i} / \sigma_{j} \rrbracket}{1-6 \eta} .
$$

Finally, using Eq. (4.21) with $m=1$, and the relations (5.21) and (5.22), we obtain the following quadratic equation for $L_{2}$ :

$$
0=Q_{0}+Q_{1} \cdot L_{2}+Q_{2} \cdot L_{2} \cdot\left(P_{0}+P_{1} \cdot L_{2}\right),
$$

where

$$
\begin{aligned}
\mathrm{P}_{0} \equiv & \frac{1}{2} \llbracket \sigma_{k} \rrbracket+2 \llbracket \eta_{i} \sigma_{i} \rrbracket+\frac{3 \llbracket \eta_{i} \sigma_{j} \rrbracket}{1-6 \eta}+\frac{12 \eta \mu_{6} \llbracket \eta_{i} \rrbracket}{\mu_{5}(1-6 \eta)}, \\
& \mathrm{P}_{1} \equiv-\frac{60}{1-6 \eta} \llbracket \eta_{i} \eta_{j} / \sigma_{j} \rrbracket-10 \llbracket \eta_{k} / \sigma_{k} \rrbracket \\
\mathrm{Q}_{0} \equiv & -\eta\left[\frac{20 \eta^{2}\left(\mu_{6} / \mu_{5}\right)^{3}}{(1-6 \eta)^{2}}+\frac{4 \eta \mu_{6} \mu_{7} / \mu_{5}^{2}}{1-6 \eta}+\frac{\mu_{8}}{8 \mu_{5}}\right] \llbracket 1 \rrbracket \\
& -\eta \frac{10 \eta\left(\mu_{6} / \mu_{5}\right)^{2}+(1-6 \eta) \mu_{7} / \mu_{5}}{(1-6 \eta)^{2}} \llbracket \sigma_{i j} \rrbracket \\
& -\frac{2 \eta \mu_{6}}{\mu_{5}(1-6 \eta)} \llbracket \sigma_{i j}^{2} \rrbracket-\frac{3 \eta}{2(1-6 \eta)} \llbracket \sigma_{i} \sigma_{j} \sigma_{i j} \rrbracket \\
& -\eta \frac{(1+24 \eta) \mu_{6}}{4 \mu_{5}(1-6 \eta)^{2}} \llbracket \sigma_{i} \sigma_{j} \rrbracket-\frac{\llbracket \sigma_{i j}^{3} \rrbracket}{3}, \\
\mathrm{Q}_{1} \equiv & \frac{10 \eta \mu_{6} / \mu_{5}}{1-6 \eta} \llbracket \eta_{j} \rrbracket+\frac{\llbracket \sigma_{k} \rrbracket}{6}+\frac{5 \llbracket \sigma_{i} \eta_{j} \rrbracket}{2(1-6 \eta)}+\frac{2}{3} \llbracket \eta_{j} \sigma_{j} \rrbracket \\
+ & \eta \frac{100 \eta\left(\mu_{6} / \mu_{5}\right)^{2}+10(1-6 \eta) \mu_{7} / \mu_{5}}{(1-6 \eta)^{2}} \llbracket \eta_{j} / \sigma_{j} \rrbracket \\
+ & \frac{25 \eta \mu_{6} / \mu_{5}}{(1-6 \eta)^{2}} \llbracket \sigma_{i} \eta_{j} / \sigma_{j} \rrbracket+\frac{5 \llbracket \sigma_{i}^{2} \eta_{j} / \sigma_{j} \rrbracket}{2(1-6 \eta)} .
\end{aligned}
$$

Due to the fact that Eq. (5.24) is quadratic in $L_{2}$, the evaluation of the structure functions is now considerably more complex than in the 3D case. In general, solutions of Eq. (5.24) must be worked out numerically.

Binary systems can be completely specified by the total packing fraction $\eta$, the concentration of one component (say $x_{2}$ ), and the diameter ratio $\sigma_{2} / \sigma_{1}$. For arbitrary and finite values of these parameters, Eq. (5.24) yields four solutions $L_{2}$ that can be obtained analytically, one of which being the physical root that verifies the convergence conditions (4.22) and (4.23).

\section{Direct correlation functions}

The knowledge of the Laplace functions $G_{i j}(s)$ allows one to obtain the direct correlation functions via Eqs. (2.11) and (3.3). Although we have not attempted a formal proof, we have checked that, in the binary case, the structural form of $\widehat{c}_{i j}(k)$ is

$$
\begin{aligned}
\widehat{c}_{i j}(k)= & \frac{1}{k^{2 d}}\left[\mathcal{P}_{i j}(i k) e^{i \sigma_{i j} k}+\mathcal{P}_{i j}(-i k) e^{-i \sigma_{i j} k}\right. \\
& \left.+\mathcal{Q}_{i j}(i k) e^{i\left(\sigma_{i}-\sigma_{j}\right) k / 2}+\mathcal{Q}_{i j}(-i k) e^{i\left(\sigma_{j}-\sigma_{i}\right) k / 2}\right],
\end{aligned}
$$

where $\mathcal{P}_{i j}(s)$ and $\mathcal{Q}_{i j}(s)$ are polynomials of degrees $(3 d-$ 1)/2 and $d-1$, respectively. Moreover, the quantity enclosed by square brackets is of order $k^{2 d}$ so that $\widehat{c}_{i j}(0)$ is finite, as required by Eq. (2.12) and enforced through Eq. (3.9). The direct correlation functions in real space, $c_{i j}(r)$ are obtained from Eq. (5.29) by means of Eq. (A2). The important point is that, upon application of the residue theorem, the structure given by Eq. (5.29) implies that $c_{i j}(r)=0$ for $r>\sigma_{i j}$ [65]. Since the RFA also complies with the physical requirement $g_{i j}(r)=0$ for $r<\sigma_{i j}$, we recover the two conditions precisely defining the PY closure to solve the OZ equation. Therefore, we find that the RFA developed in this paper yields the PY solution for hard-hypersphere mixtures of odd dimensions. This is a remarkable result since both approaches (RFA and PY) are, in principle, rather independent.

\section{RESULTS FOR 5D BINARY MIXTURES}

Here, we consider a mixture of two types $(i=1,2)$ of 5D spheres with arbitrary diameters $\sigma_{i}$ and concentrations $x_{i}$. For simplicity, we fix $\sigma_{1}=1$.

Figure 3 compares the compressibility factors calculated from the virial $\left(Z_{v}\right)$ and compressibility $\left(Z_{c}\right)$ routes [cf. Eqs. (4.24) and (4.27), respectively] with those from computer simulations (molecular dynamics) [40] and a semiempirical equation of state [34] for a mixture with a diameter ratio $\sigma_{2} / \sigma_{1}=\frac{1}{4}$ and mole fractions $x_{2}=\frac{1}{4}, \frac{1}{2}$, and $\frac{3}{4}$. We observe that $Z_{c}$ and $Z_{v}$ bound both the simulation data and the proposal of Ref. [34], with $Z_{c}$ slightly above and $Z_{v}$ slightly below. The agreement is very good at low densities (packing fraction lower than 0.1 ) and reasonably good over the whole density range $(0 \leqslant \eta<0.19)$ of fluid phase predicted for the one-component system. In general, the compressibility route gives better results than the virial one. A similar relation $Z_{v}<Z<Z_{c}$, with $Z$ being the actual compressibility factor, has been observed for the PY solution of the pure 5D system [65]. Figure 3 also includes the interpolation formula $Z=\frac{2}{5} Z_{v}+\frac{3}{5} Z_{c}$ [38]. This Carnahan-Starling-like recipe presents very good agreement with simulation data and is practically indistinguishable from the semiempirical equation of state proposed in Ref. [34], except for $x_{2}=\frac{3}{4}$ and $\eta>0.15$. Unfortunately, no simulation data are available in those cases.

Next, it is instructive to examine the size ratio dependence of the compressibility factor. Figure 4 compares values of $Z_{v}$ obtained from the RFA-PY solution via the virial route with those calculated from the analytical expression given in Ref. [34]. These evaluations correspond to a packing fraction $\eta=0.15$ and a set of molar fractions ranging from $x_{2}=\frac{1}{32}$ 


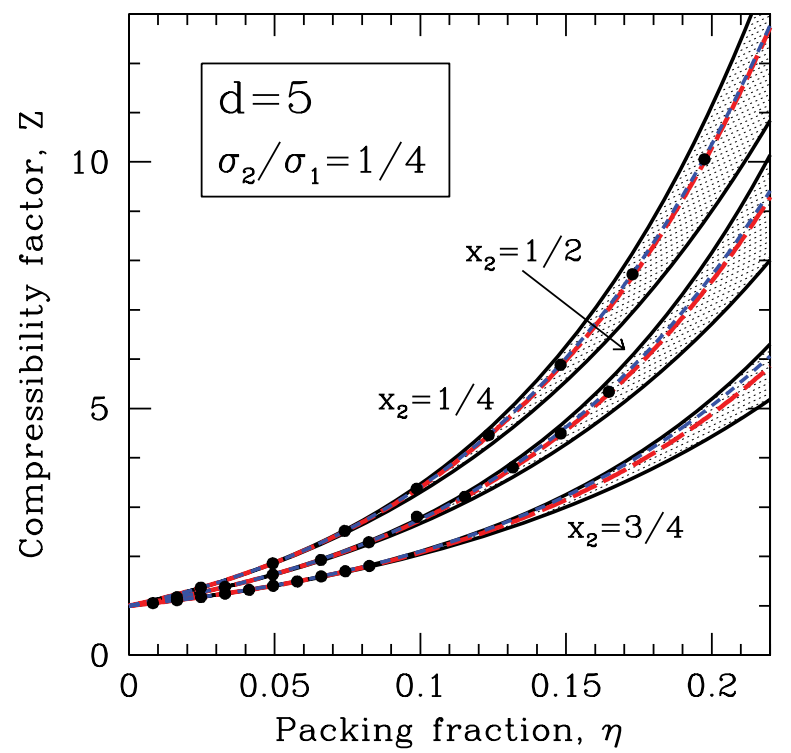

FIG. 3. (Color online) Compressibility factor as a function of the packing fraction $\eta$ for a binary mixture at $d=5$ with $\sigma_{2} / \sigma_{1}=\frac{1}{4}$ and mole fractions $x_{2}=\frac{1}{4}, \frac{1}{2}$, and $\frac{3}{4}$ (indicated on the plot). RFAPY results obtained from the virial and the compressibility routes (solid lines on the right and left of the shaded area, respectively) are compared with predictions of an analytical equation proposed in Ref. [34] (short dashed lines) and simulation results from Ref. [40] (symbols). The long dashed lines represent the interpolation $Z=$ $\frac{2}{5} Z_{v}+\frac{3}{5} Z_{c}$.

to $x_{2}=\frac{1}{4}$. As the concentration $x_{2}$ decreases, we find, as expected, that the compressibility factor comes to have a weaker dependence on $\sigma_{2}$. The general trends of $Z_{v}$ versus $\sigma_{2}$ for different mole fractions $x_{2}$ qualitatively agree with the predictions of the equation of state proposed in Ref. [34] (there are no simulation data available for this analysis). The differences between both calculations (at most 9\%) are similar to those observed before.

Now, we analyze the structure and correlation functions. Instead of the conventional structure factors $S_{i j}$, we consider some combinations of them, which may easily be associated with fluctuations of the thermodynamic variables [91,93]:

$$
\begin{gathered}
S_{N N}(k)=S_{11}(k)+S_{22}(k)+2 S_{12}(k), \\
S_{N c}(k)=x_{2} S_{11}(k)-x_{1} S_{22}(k)+\left(x_{2}-x_{1}\right) S_{12}(k), \\
S_{c c}(k)=x_{2}^{2} S_{11}(k)+x_{1}^{2} S_{22}(k)-2 x_{1} x_{2} S_{12}(k) .
\end{gathered}
$$

In the limit of small wave number $(k \rightarrow 0), S_{N N}$ and $S_{c c}$ become the mean square fluctuations in the particle number and concentration, respectively, whereas $S_{N c}$ is the correlation between these two fluctuations.

In Fig. 5, we plot the number-concentration structure factors for a packing fraction $\eta=0.15$, a diameter ratio $\sigma_{2} / \sigma_{1}=\frac{1}{4}$, and decreasing values of the particle- 2 concentration, $x_{2}=\frac{1}{2}$, $\frac{1}{4}, \frac{1}{8}, \frac{1}{16}$, and $\frac{1}{32}$. As expected, $S_{c c}$ and $S_{N c}$ reduce smoothly to zero with $x_{2}$ for all wave numbers, whereas the number-number structure factor $S_{N N}$ converges to the static structure factor of the one-component fluid in this limit (dashed line). Notice that $S_{N N}$ and $S_{c c}$ are positive for all $k$ by definition. The main modifications of the structure factor $S_{N N}$ with increasing $x_{2}$

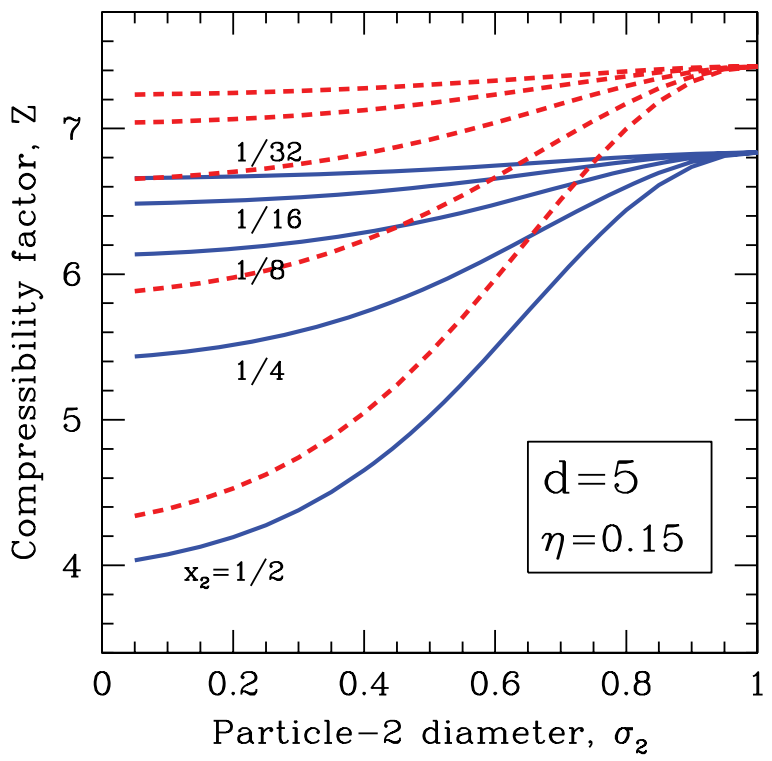

FIG. 4. (Color online) Compressibility factor obtained from the RFA-PY method for a binary mixture at $d=5$, as a function of the particle diameter $\sigma_{2}\left(\sigma_{1}=1\right)$ for $\eta=0.15$ and mole fractions $x_{2}$ indicated on the plot (solid lines). Predictions of the analytical equation from Ref. [34] are shown with dashed lines.

are the variation of height of the main peak, with its position very weakly altered, and an increase of the values at short $k$.

Evaluations of the direct correlation functions defined (in the Fourier space) by the OZ equation (2.10) are shown in Fig. 6. The results correspond to an equimolar mixture for different diameter ratios from $\sigma_{2} / \sigma_{1}=\frac{1}{2}$ to nearly equivalent hypersphere sizes $\sigma_{2} / \sigma_{1}=\frac{31}{32}$. In the limit $\sigma_{2} / \sigma_{1} \rightarrow 1$, the

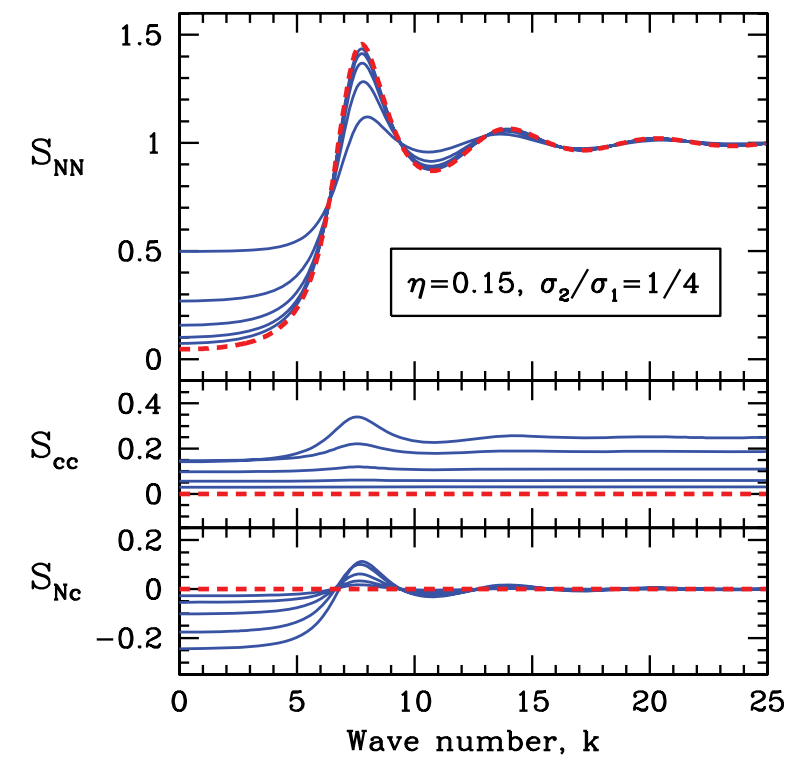

FIG. 5. (Color online) Number-concentration structure factors $S_{N N}, S_{N c}$, and $S_{c c}$, as obtained from the RFA-PY method for a binary mixture in $d=5$ dimensions with diameter ratio $\sigma_{2} / \sigma_{1}=\frac{1}{4}$, packing fraction $\eta=0.15$, and mole fractions $x_{2}=\frac{1}{2}, \frac{1}{4}, \frac{1}{8}, \frac{1}{16}$, and $\frac{1}{32}$ (solid lines). As $x_{2}$ is reduced, the curves converge to the solution of the pure fluid (dashed lines). 


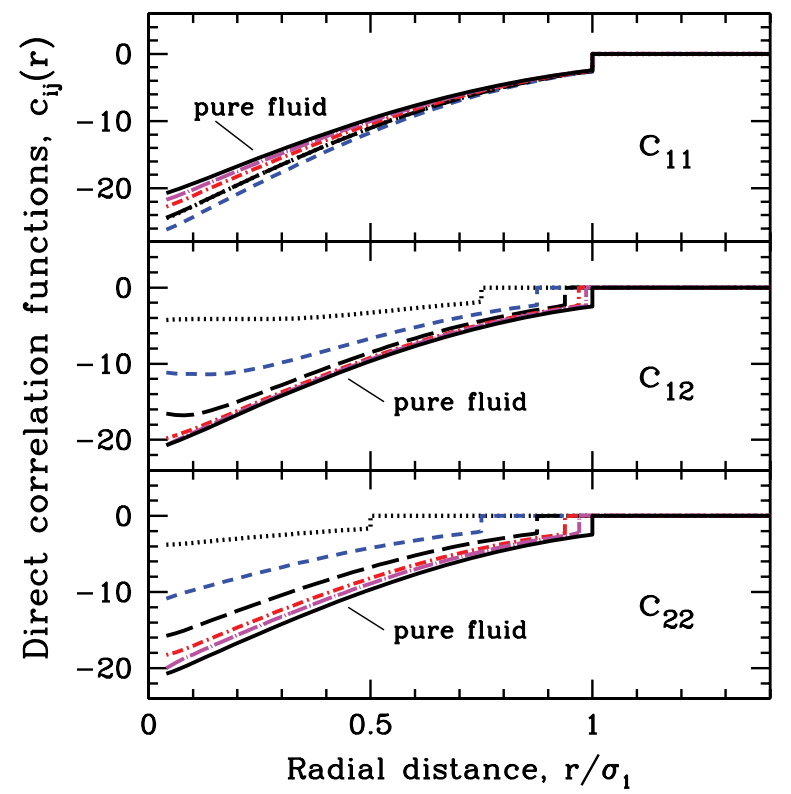

FIG. 6. (Color online) Direct correlation functions obtained from the RFA-PY method for an equimolar $\left(x_{1}=x_{2}=\frac{1}{2}\right)$ binary mixture in $d=5$ dimensions at $\eta=0.15$ and various diameter ratios, $\sigma_{2} / \sigma_{1}=$ $\frac{1}{2}$ (dotted line), $\frac{3}{4}$ (dashed line), $\frac{7}{8}$ (long dashed line), $\frac{15}{16}$ (dasheddotted line), and $\frac{31}{32}$ (long dashed-dotted line). The pure-fluid solution is displayed for comparison (solid line).

three correlation functions $c_{i j}(r)$ become identical and match the pure-fluid values. Figure 6 clearly shows that the direct correlation functions $c_{i j}(r)$ vanish for $r>\sigma_{i j}$, thus, confirming that the RFA method yields solutions of the PY closure to the $\mathrm{OZ}$ equation, as discussed in Sec. V C.

Figures 7 and 8 show the radial distribution functions $g_{i j}(r)$ of a highly asymmetric binary 5D-sphere mixture with $\sigma_{2} / \sigma_{1}=\frac{1}{4}$ and $x_{2}=\frac{1}{4}$, at the reduced densities $\rho \sigma_{1}^{5}=1.4$ and $\rho \sigma_{1}^{5}=2.0$, respectively. The selected densities lie in the

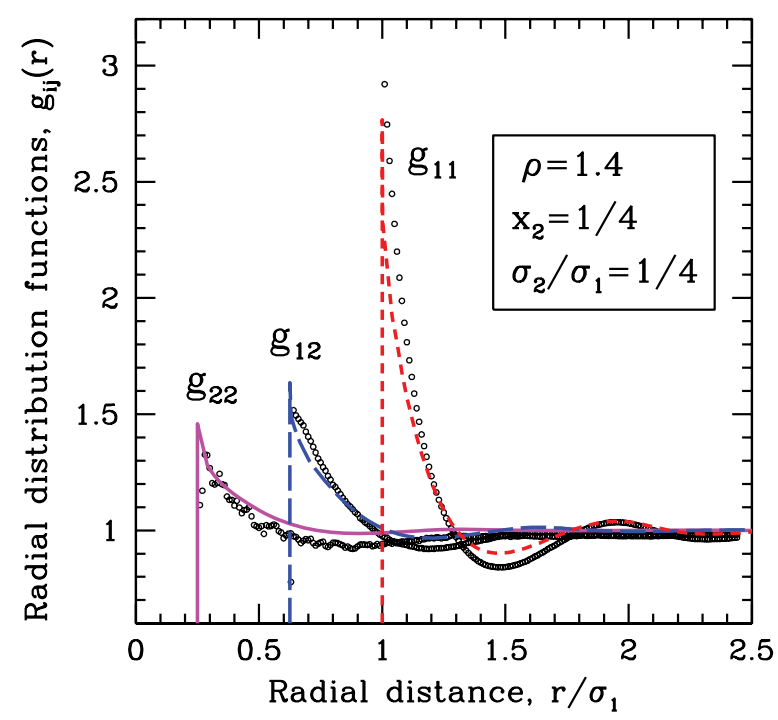

FIG. 7. (Color online) Radial distribution functions for a binary mixture in $d=5$ dimensions with parameters $x_{2}=\frac{1}{4}, \sigma_{2} / \sigma_{1}=\frac{1}{4}$, and density $\rho \sigma_{1}^{5}=1.4(\eta=0.172774)$. (Solid lines) Results from the RFA-PY method. (Symbols) Computer simulations [40].

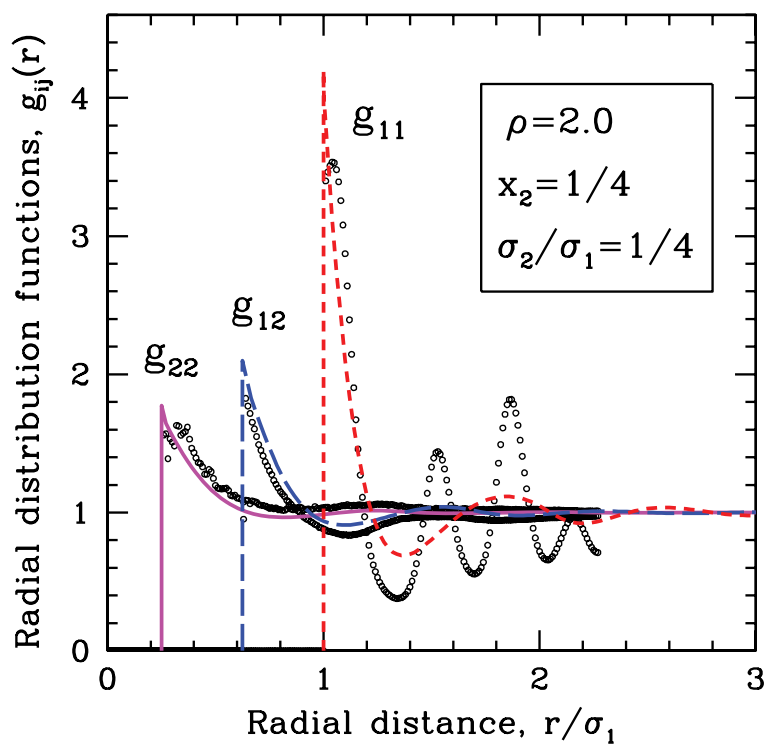

FIG. 8. (Color online) As in Fig. 7, but for $\rho \sigma_{1}^{5}=2.0(\eta=$ $0.246820)$.

proximity of the phase transition for the pure one-component fluid $\left(\eta_{f}=0.19\right)$ predicted by Michels and Trappeniers [6]. We see that the RFA-PY solution at $\eta \approx 0.17$ gives reasonable good values for all pair functions throughout the $r$ region (see Fig. 7). At a higher packing fraction $\eta \approx 0.25$, we observe, in Fig. 8, that similar quantitative trends in $g_{22}$ and $g_{12}$ are followed by both simulations and RFA-PY solutions. However, there are discrepancies particularly severe in the values of $g_{11}$ corresponding to pairs of big particles. The strong oscillations observed in the simulation data of $g_{11}$ can be considered as a signature of a solid phase at this density [40], which is not captured by the RFA-PY solution.

\section{CONCLUSIONS}

In this paper, we have extended the RFA method to multicomponent systems of hard hyperspheres in odd dimensions. The main features of this approach are based on simple physical considerations on Laplace functionals $G_{i j}(s)$ of the radial distribution functions, which are closely related to the structure functions of the fluid. The basic physical requirements upon which the method is based are: (i) The radial distribution functions take finite values at contact and vanish inside the core, which implies that $\lim _{s \rightarrow \infty}\left[s^{(5-d) / 2} e^{\sigma_{i j} s} G_{i j}(s)\right]=$ finite; (ii) the isothermal compressibility is finite, which implies $\lim _{s \rightarrow 0}\left[G_{i j}(s)-(d-2) ! ! / s^{2}\right]=$ finite; and (iii) the first three terms in the series expansion in powers of density of the structure factors are exact. Condition (iii) involves the evaluation of the overlap volume of two arbitrary $d$ spheres. For space of odd dimensionality, we have found the exact, analytical, and closed-form expression for the overlap volume of two hyperspheres of arbitrary sizes as a function of the center distance [cf. Eqs. (B10) and (B16)]. We have been able to perform this evaluation thanks to the use of reverse Bessel polynomials and Fourier analysis.

The primary result of the present paper has been to provide a theoretical method for the evaluation of thermodynamic and structural quantities of multicomponent mixtures of hard 
hyperspheres at odd dimensions. We have shown that this approach gives the exact solution of the OZ equation with the PY closure. From that perspective, our paper extends, on one hand, the Lebowitz solution for 3D-sphere mixtures [80] to higher dimensions and, on the other hand, the PY solution for one-component hyperspheres $[3,7,45,62]$ to the case of mixtures.

Although the theory here developed is general and applies to any odd-dimensional hard-hypersphere fluid with an arbitrary number of components, the results in this paper concentrate on $5 \mathrm{D}$ binary mixtures. We have checked that the virial $\left(Z_{v}\right)$ and compressibility $\left(Z_{c}\right)$ routes to the compressibility factor under- and overestimate, respectively, the simulation data, the interpolation approximation $Z=\frac{2}{5} Z_{v}+\frac{3}{5} Z_{c}$ providing excellent results. In addition, reasonable agreement between RFA-PY results and simulation data for the radial distribution functions are found at densities lower than that of the fluid-solid phase transition predicted for a pure fluid at this dimension.

The implementation of the RFA method developed here involves as many unknowns as the minimum number required by the physical conditions and that is why it coincides with the PY solution. However, as done for 3D mixtures [81] and for $d$-dimensional one-component systems [65], one can go beyond the PY level by adding an extra matricial term $L_{n+2}$ in Eqs. (4.2) and (4.3), and by replacing I with $(1+u s) I$ in Eq. (4.3). The elements of $L_{n+2}$ and the parameter $u$ are free and can be fixed, for instance, by imposing given expressions for the contact values $g_{i j}\left(\sigma_{i j}^{+}\right)$and the thermodynamically consistent isothermal susceptibility $\chi$.

We expect that the results presented in this paper can stimulate simulation studies on multicomponent systems of hard hyperspheres. We also plan to undertake the investigation of possible fluid-fluid demixing transitions predicted by the equations of state obtained here within the RFA-PY approach.

\section{ACKNOWLEDGMENTS}

We would like to thank M. González-Melchor, who kindly sent us her simulation data, which were used in Figs. 7 and 8. The work of R.D.R. has been supported by the Consejo Nacional de Investigaciones Científicas y Técnicas (CONICET, Argentina) through Grant No. PIP 112-20080101474. A.S. acknowledges support from the Ministerio de Ciencia e Innovación (Spain) through Grant No. FIS201016587, partially financed by FEDER (Fondo Europeo de Desarrollo Regional) funds.

\section{APPENDIX A: FOURIER TRANSFORM}

In odd-dimensional spaces, the Fourier transform of an arbitrary radial function $\mu(r)$ and the inverse operation can be evaluated in terms of the reverse Bessel polynomial $\theta_{n}(t)$ of degree $n=(d-3) / 2$ as [65]

$$
\begin{gathered}
\widehat{\mu}(k)=\frac{(2 \pi)^{(d-1) / 2}}{k^{d-2}} i \int_{-\infty}^{\infty} d r r \mu(r) \theta_{n}(i k r) e^{-i k r}, \\
\mu(r)=\frac{(2 \pi)^{-(d+1) / 2}}{r^{d-2}} i \int_{-\infty}^{\infty} d k k \widehat{\mu}(k) \theta_{n}(i k r) e^{-i k r} .
\end{gathered}
$$

Alternatively,

$$
\begin{gathered}
\widehat{\mu}(k)=\frac{(2 \pi)^{(d-1) / 2}}{k^{d-2}} 2 \operatorname{Im}\left\{\mathcal{F}_{n}[\mu(r)](-i k)\right\}, \\
\mu(r)=\frac{(2 \pi)^{-(d+1) / 2}}{r^{d-2}} 2 \operatorname{Im}\left\{\mathcal{F}_{n}[\widehat{\mu}(k)](-i r)\right\},
\end{gathered}
$$

where $\mathcal{F}_{n}$ is a Laplace functional defined by

$$
\mathcal{F}_{n}[\mu(r)](s) \equiv \int_{0}^{\infty} d x x \mu(x) \theta_{n}(s x) e^{-s x} .
$$

From an integral relation on $\theta_{n}(t)$, one arrives at [65]

$$
\begin{gathered}
\mathcal{F}_{n}[1](s)=\frac{\theta_{n+1}(0)}{s^{2}}=\frac{(2 n+1) ! !}{s^{2}}, \\
\mathcal{F}_{n}[\Theta(r-a)](s)=\frac{\theta_{n+1}(a s) e^{-a s}}{s^{2}} .
\end{gathered}
$$

\section{APPENDIX B: COVOLUME OF TWO HYPERSPHERES}

The overlap volume of two hyperspheres of radii $a$ and $b$, whose centers are a distance $r=|\boldsymbol{r}|$, can be evaluated as

$$
\Omega_{a, b}(r)=\int d \boldsymbol{r}^{\prime} \Theta\left(a-\left|\boldsymbol{r}^{\prime}\right|\right) \Theta\left(b-\left|\boldsymbol{r}^{\prime}-\boldsymbol{r}\right|\right) .
$$

Henceforth, without loss of generality, we assume $a \leqslant b$. The right-hand side of Eq. (B1) is a convolution. Thus, in Fourier space, Eq. (B1) reads

$$
\widehat{\Omega}_{a, b}(k)=\widehat{\Theta}_{a}(k) \widehat{\Theta}_{b}(k)
$$

where we have called

$$
\widehat{\Theta}_{a}(k) \equiv \int d \mathbf{r} \Theta(a-r) e^{-i \mathbf{k} \cdot \mathbf{r}} .
$$

Now using Eqs. (A3), (A6), and (A7), and taking into account that $\Theta(a-r)=1-\Theta(r-a)$, we get

$$
\begin{aligned}
\widehat{\Theta}_{a}(k) & =\frac{(2 \pi)^{(d-1) / 2}}{k^{d-2}} 2 \operatorname{Im}\left[\frac{(2 n+1) ! !}{s^{2}}-\frac{\theta_{n+1}(a s) e^{-a s}}{s^{2}}\right]_{s=-\mathrm{i} k} \\
& =\frac{(2 \pi)^{(d-1) / 2}}{k^{d}} i\left[\theta_{n+1}(-i k a) e^{i k a}-\theta_{n+1}(i k a) e^{-i k a}\right] .
\end{aligned}
$$

Therefore,

$$
\begin{aligned}
\widehat{\Omega}_{a, b}(k)= & \frac{(2 \pi)^{d-1}}{k^{2 d}}\left[\theta_{n+1}(i k a) \theta_{n+1}(-i k b) e^{i k(b-a)}\right. \\
& +\theta_{n+1}(-i k a) \theta_{n+1}(i k b) e^{-i k(b-a)} \\
& -\theta_{n+1}(-i k a) \theta_{n+1}(-i k b) e^{i k(b+a)} \\
& \left.-\theta_{n+1}(i k a) \theta_{n+1}(i k b) e^{-i k(b+a)}\right] .
\end{aligned}
$$

We can obtain $\Omega_{a, b}(r)$ using the inverse Fourier transform (A2). Integration on the complex plane and the application of the residue theorem yield

$$
\begin{aligned}
\Omega_{a, b}(r)= & \frac{(2 \pi)^{(d-1) / 2}}{r^{d-2}}\left[R_{4 n+4}^{(a, b)}(r) \Theta(b+a-r)\right. \\
& \left.-R_{4 n+4}^{(-a, b)}(r) \Theta(b-a-r)\right],
\end{aligned}
$$


with

$$
\begin{aligned}
R_{4 n+4}^{( \pm a, b)}(r)= & \operatorname{Res}_{t=0}\left[\frac{\theta_{n+1}(\mp t a) \theta_{n+1}(-t b) \theta_{n}(t r) e^{t(b \pm a-r)}}{t^{4 n+5}}\right] \\
= & \frac{1}{(4 n+4) !}\left[\partial_{t}^{4 n+4} \theta_{n+1}(\mp t a) \theta_{n+1}(-t b)\right. \\
& \left.\times \theta_{n}(t r) e^{t(b \pm a-r)}\right]_{t=0} .
\end{aligned}
$$

Equation (B7) implies that $R_{4 n+4}^{( \pm a, b)}(r)$ are polynomials of degree $4 n+4=2 d-2$. If $r<b-a$, the smaller hypersphere will be fully contained within the bigger one so that $\Omega_{a, b}(r)$ will be equivalent to the volume of the former, i.e.,

$$
\Omega_{a, b}(r)=(2 a)^{d} v_{d}=\frac{2 a^{d}(2 \pi)^{(d-1) / 2}}{d ! !}, \quad r \leqslant b-a .
$$

Thus, according to Eq. (B6), the difference between the polynomials $R_{4 n+4}^{( \pm a, b)}$ takes the following expression (for any $r)$ :

$$
R_{4 n+4}^{(a, b)}(r)-R_{4 n+4}^{(-a, b)}(r)=\frac{2 a^{2 n+3} r^{2 n+1}}{(2 n+3) ! !} .
$$

Therefore, the final result is

$$
\Omega_{a, b}(r)= \begin{cases}\frac{(2 \pi)^{(d-1) / 2}}{d ! !} 2 a^{d}, & r \leqslant b-a, \\ (2 \pi)^{(d-1) / 2} \frac{R_{4 n+4}^{(a, b)}(r)}{r^{d-2}}, & b-a \leqslant r \leqslant b+a, \\ 0, & r \geqslant b+a .\end{cases}
$$

Let us now obtain an explicit expression for the polynomial $R_{4 n+4}^{(a, b)}(r)$. According to Eq. (B7), it is the coefficient of $t^{4 n+4}$ in the expansion of $\theta_{n+1}(-t a) \theta_{n+1}(-t b) \theta_{n}(t r) e^{t(b+a-r)}$ in powers of $t$. First, note that

$$
\theta_{n+1}(-t a) \theta_{n+1}(-t b)=\sum_{\ell=0}^{2 n+2} c_{n, \ell}^{(a, b)} t^{\ell}
$$

where

$$
c_{n, \ell}^{(a, b)}=(-1)^{\ell} \sum_{\ell_{1}=\max (0, \ell-n-1)}^{\min (\ell, n+1)} \omega_{n+1, \ell_{1}} \omega_{n+1, \ell-\ell_{1}} a^{\ell_{1}} b^{\ell-\ell_{1}} .
$$

Next,

$$
\theta_{n+1}(-t a) \theta_{n+1}(-t b) \theta_{n}(t r)=\sum_{\ell=0}^{3 n+2} d_{n, \ell}^{(a, b)}(r) t^{\ell},
$$

with

$$
d_{n, \ell}^{(a, b)}(r)=\sum_{\ell_{1}=\max (0, \ell-2 n-2)}^{\min (\ell, n)} \omega_{n, \ell_{1}} c_{n, \ell-\ell_{1}}^{(a, b)} r^{\ell_{1}}
$$

Finally, the coefficient of $t^{\ell}$ in the $t$ expansion of $\theta_{n+1}(-t a) \theta_{n+1}(-t b) \theta_{n}(t r) e^{t(b+a-r)}$ is

$$
\sum_{\ell_{1}=\max (0, \ell-3 n-2)}^{\ell} \frac{(a+b-r)^{\ell_{1}}}{\ell_{1} !} d_{n, \ell-\ell_{1}}^{(a, b)}(r) .
$$

Setting $\ell=4 n+4$ and inserting Eq. (B14), one obtains

$$
\begin{aligned}
R_{4 n+4}^{(a, b)}(r)= & (a+b-r)^{n+2} \sum_{\ell=0}^{3 n+2} \frac{(a+b-r)^{3 n+2-\ell}}{(4 n+4-\ell) !} \\
& \times \sum_{\ell_{1}=\max (0, \ell-2 n-2)}^{\min (\ell, n)} \omega_{n, \ell_{1}} c_{n, \ell-\ell_{1}}^{(a, b)} r^{\ell_{1}} \cdot
\end{aligned}
$$

Thus, for example, at $d=3,5$ (respectively, $n=0,1$ ) one finds

$$
R_{4}^{(a, b)}(r)=\frac{(a+b-r)^{2}}{24}\left[r^{2}+2(a+b) r-3(b-a)^{2}\right],
$$

$$
\begin{aligned}
R_{8}^{(a, b)}(r)= & \frac{(a+b-r)^{3}}{1920}\left[3 r^{5}+9(a+b) r^{4}\right. \\
& -2\left(a^{2}-18 a b+b^{2}\right) r^{3}-2(b-a)^{2}(a+b) r^{2} \\
& \left.+15(b-a)^{4} r+5(b-a)^{4}(a+b)\right] . \quad(\mathrm{B} 18)
\end{aligned}
$$

It can be checked that $R_{4 n+4}^{(a, b)}(r)$ admits the following structure:

$$
\begin{aligned}
R_{4 n+4}^{(a, b)}(r)= & (a+b-r)^{n+2}\left[r^{2 n+1} P_{n+1}^{(a, b)}(r)\right. \\
& \left.+(b-a)^{2} K_{2 n}^{(a, b)}(r)\right],
\end{aligned}
$$

where $P_{n+1}^{(a, b)}(r)$ and $K_{2 n}^{(a, b)}(r)$ are polynomials of degree $n+1$ and $2 n$, respectively. Clearly, in the case of identical hyperspheres $(a=b), R_{4 n+4}^{(a, a)}(r)$ adopts the known expression

$$
R_{4 n+4}^{(a, a)}(r)=(2 a-r)^{n+2} r^{2 n+1} P_{n+1}^{(a, a)}(r),
$$

with $P_{n+1}^{(1,1)}(r)$ given by Eqs. (B7) and (B8) of Ref. [65].

\section{APPENDIX C: PROPERTIES OF B $(s)$}

The series expansion of the function $\phi(x)$ defined by Eq. (4.5) is

$$
\phi_{m}(x)=-\sum_{\ell=1}^{\infty} \frac{(-1)^{m+\ell}}{(m+\ell) !} x^{\ell} .
$$

Therefore, Eq. (4.4) yields

$$
\Phi_{m}(s)=\sum_{\ell=1-\delta}^{\infty} \mathrm{C}_{d+\ell-m} s^{\ell} \quad(\delta=0,1),
$$

where the diagonal matrices $\mathrm{C}_{m}$ are

$$
\left(\mathrm{C}_{m}\right)_{i i}=-(-2 \pi)^{(d-1) / 2} x_{i} \frac{\left(-\sigma_{i}\right)^{m}}{m !} .
$$

Thus, the series expansion of the matrix $\mathrm{B}(s)$ defined by Eq. (4.3) is

$$
\mathrm{B}(s)=\sum_{\ell=0}^{\infty} \mathrm{B}_{\ell} s^{\ell}
$$

with

$$
\begin{array}{r}
\mathrm{B}_{0}= \begin{cases}\mathrm{I} & (\delta=0), \\
\mathrm{I}+\rho \sum_{m=0}^{n+1} \mathrm{C}_{d-m} \cdot \mathrm{L}_{m} & (\delta=1),\end{cases} \\
\mathrm{B}_{\ell}=\rho \sum_{m=0}^{n+1} \mathrm{C}_{d+\ell-m} \cdot \mathrm{L}_{m} \quad(\ell \geqslant 1) .
\end{array}
$$


According to Eqs. (C2) and (C4),

$$
\begin{gathered}
\lim _{s \rightarrow 0} \Phi_{m}(s)= \begin{cases}0 & (\delta=0), \\
\mathrm{C}_{d-m} & (\delta=1),\end{cases} \\
\lim _{s \rightarrow 0} \mathrm{~B}(s)= \begin{cases}\mathrm{I} & (\delta=0), \\
\mathrm{I}+\rho \sum_{m=0}^{n+1} \mathrm{C}_{d-m} \cdot \mathrm{L}_{m} & (\delta=1) .\end{cases}
\end{gathered}
$$

As for the behaviors in the limit $s \rightarrow \infty$, it is straightforward from Eqs. (4.3)-(4.5) to get

$$
\begin{gathered}
\lim _{x \rightarrow \infty} \phi_{m}(x)=\frac{(-1)^{m}}{m !}, \\
\lim _{s \rightarrow \infty} \Phi_{m}(s)= \begin{cases}-\mathrm{C}_{d-m} & (\delta=0), \\
0 & (\delta=1),\end{cases} \\
\lim _{s \rightarrow \infty} \mathrm{B}(s)= \begin{cases}1-\rho \sum_{m=0}^{n+1} \mathrm{C}_{d-m} \cdot \mathrm{L}_{m} & (\delta=0), \\
\mathrm{l} & (\delta=1) .\end{cases}
\end{gathered}
$$

\section{APPENDIX D: THE RATIONAL FUNCTION APPROXIMATION IN THE LOW-DENSITY LIMIT}

Let us consider the low-density expansions of $\mathrm{L}_{m}$ and $\mathrm{B}(s)$,

$$
\begin{gathered}
\mathrm{L}_{m}=\mathrm{L}_{m}^{(0)}+\rho \mathrm{L}_{m}^{(1)}+O\left(\rho^{2}\right), \\
\mathrm{B}(s)=\mathrm{I}+\rho \mathrm{B}^{(1)}(s)+O\left(\rho^{2}\right),
\end{gathered}
$$

where

$$
B_{i j}^{(1)}(s)=\sum_{m=0}^{n+1}\left[\Phi_{m}(s)\right]_{i i}\left[\mathrm{~L}_{m}^{(0)}\right]_{i j} .
$$

Insertion into Eq. (4.1) yields Eq. (3.13) with

$$
\begin{gathered}
G_{i j}^{(0)}(s)=\frac{e^{-\sigma_{i j} s}}{s^{2}} \sum_{m=0}^{n+1}\left[\mathrm{~L}_{m}^{(0)}\right]_{i j} s^{m}, \\
G_{i j}^{(1)}(s)=\frac{e^{-\sigma_{i j} s}}{s^{2}} \sum_{m=0}^{n+1}\left[\mathrm{~L}_{m}^{(1)}-\mathrm{L}_{m}^{(0)} \cdot \mathrm{B}^{(1)}(s)\right]_{i j} s^{m} .
\end{gathered}
$$

Comparison between Eqs. (3.14) and Eq. (D4) implies that

$$
\left[\mathrm{L}_{m}^{(0)}\right]_{i j}=\omega_{n+1, m} \sigma_{i j}^{m} .
$$

Now, let us consider the matrices $\mathrm{L}_{m}^{(1)}$. It is convenient to express their elements as

$$
\left[\mathrm{L}_{m}^{(1)}\right]_{i j}=\sum_{\ell=1}^{\mathcal{N}} x_{\ell} L_{m, i j \ell}^{(1)} .
$$

Using Eqs. (4.4) and (4.5), one can recast Eq. (D5) into the form (3.15) with

$$
\begin{aligned}
G_{i j \ell}^{(1)}(s)= & \frac{e^{-\sigma_{i j} s}}{s^{2}} \sum_{m=0}^{n+1} L_{m, i j \ell}^{(1)} s^{m}+\frac{v_{d}}{s^{d-2}} G_{i \ell}^{(0)}(s) G_{j \ell}^{(0)}(s) \\
& -v_{d} \frac{e^{-\sigma_{i j} s}}{s^{d+2}} \theta_{n+1}\left(\sigma_{i \ell} s\right) \sum_{m=0}^{n+1} \omega_{n+1, m}\left(\sigma_{j \ell} s\right)^{m} \\
& \times \sum_{q=0}^{d-m-\delta} \frac{\left(-\sigma_{\ell} s\right)^{q}}{q !}
\end{aligned}
$$

Comparison with Eqs. (3.18) and (3.19) allows us to identify

$$
\begin{gathered}
\sum_{m=0}^{n+1} L_{m, i j \ell}^{(1)} s^{m}=v_{d} s \bar{Q}_{i j \ell}(s) \quad(\delta=0), \\
\sum_{m=0}^{n+1} L_{m, i j \ell}^{(1)} s^{m}=v_{d} s \bar{Q}_{i j \ell}(s)-v_{d} \theta_{n+1}\left(\sigma_{i \ell} s\right) \sum_{m=0}^{n+1} \frac{\omega_{n+1, m}}{(d-m) !} \\
\times \sigma_{j \ell}^{m}\left(-\sigma_{\ell}\right)^{d-m} \quad(\delta=1) .
\end{gathered}
$$

Therefore, the RFA proposal (4.1)-(4.5) is consistent with the exact result to first order in density.

\section{APPENDIX E: PURE-FLUID LIMIT}

Here, we consider $\sigma_{i j}=\sigma=1 \forall i, j$, within the case $\delta=$ 0 . Then, $\rho \Phi_{m}(s)=\lambda_{d} \phi_{d-m}(s) \llbracket \eta_{k} \rrbracket$, and one can expect that $\mathrm{L}_{m}=a_{m}[1]$, with $a_{m}$ being a certain constant. Here, we use the matrix notation defined in Eq. (5.1) and have defined

$$
\lambda_{d} \equiv \frac{v_{d}}{v_{d}}=(-1)^{(d-1) / 2} 2^{d-1} d ! ! .
$$

Thus,

$$
\mathrm{L}(s)=\sum_{m} a_{m} s^{m} \llbracket 1 \rrbracket,
$$

and

$$
\mathrm{B}(s)=\mathrm{I}+\lambda_{d} \sum_{m=0}^{n+1} \phi_{d-m}(s) a_{m} \llbracket \eta_{i} \rrbracket .
$$

Using the mathematical property (F1) and taking into account that $\operatorname{tr}\left(\llbracket \eta_{i} \rrbracket\right)=\eta$,

$$
\mathrm{B}^{-1}(s)=\mathrm{I}-\lambda_{d} \frac{\sum_{m=0}^{n+1} \phi_{d-m}(s) a_{m}}{1+\lambda_{d} \eta \sum_{m=0}^{n+1} \phi_{d-m}(s) a_{m}} \llbracket \eta_{i} \rrbracket .
$$

From Eqs. (E2) and (E4), it readily follows:

$$
\mathrm{L}(s) \cdot \mathrm{B}^{-1}(s)=\frac{\sum_{m=0}^{n+1} a_{m} s^{m}}{1+\lambda_{d} \eta \sum_{m=0}^{n+1} \phi_{d-m}(s) a_{m}} \llbracket 1 \rrbracket .
$$

Finally, Eq. (4.1) becomes

$$
G_{i j}(s)=\frac{e^{-s}}{s^{2}} \frac{\sum_{m} a_{m} s^{m}}{1+\lambda_{d} \eta \sum_{m=0}^{n+1} \phi_{d-m}(s) a_{m}}, \quad \forall i, j .
$$

This coincides with the PY solution of the one-component fluid in $d=2 n+3$ dimensions, as derived from the RFA in Ref. [65].

\section{APPENDIX F: INVERSION OF MATRICES}

Let us consider square matrices defined following the notation of Eq. (5.1). Then, as proved in the following, one has

$$
\begin{gathered}
\left(\mathrm{I}+\llbracket A_{j} \rrbracket\right)^{-1}=\mathrm{I}-\frac{\llbracket A_{j} \rrbracket}{1+a}, \quad\left(\mathrm{I}+\llbracket A_{i} \rrbracket\right)^{-1}=\mathrm{I}-\frac{\llbracket A_{i} \rrbracket}{1+a}, \\
\left(\mathrm{I}+\llbracket C_{i} B_{j} \rrbracket\right)^{-1}=\mathrm{I}-\frac{\llbracket C_{i} B_{j} \rrbracket}{1+\beta}, \\
\left(\mathrm{I}+\llbracket B_{i} C_{j} \rrbracket\right)^{-1}=\mathrm{I}-\frac{\llbracket B_{i} C_{j} \rrbracket}{1+\beta} .
\end{gathered}
$$




$$
\begin{aligned}
\left(\mathrm{I}+\llbracket A_{j} \rrbracket+\llbracket C_{i} B_{j} \rrbracket\right)^{-1}= & \mathrm{I}+\frac{1}{\gamma}\left\{\alpha \llbracket B_{j} \rrbracket-(1+a) \llbracket C_{i} B_{j} \rrbracket\right. \\
& \left.-(1+\beta) \llbracket A_{j} \rrbracket+b \llbracket C_{i} A_{j} \rrbracket\right\},
\end{aligned}
$$

$$
\begin{aligned}
\left(\mathrm{I}+\llbracket A_{i} \rrbracket+\llbracket B_{i} C_{j} \rrbracket\right)^{-1}= & \mathrm{I} \\
& +\frac{1}{\gamma}\left\{\alpha \llbracket B_{i} \rrbracket-(1+a) \llbracket B_{i} C_{j} \rrbracket\right. \\
& \left.-(1+\beta) \llbracket A_{i} \rrbracket+b \llbracket A_{i} C_{j} \rrbracket\right\},
\end{aligned}
$$

where

$$
a \equiv \operatorname{tr}\left(\llbracket A_{j} \rrbracket\right)=\operatorname{tr}\left(\llbracket A_{i} \rrbracket\right), \quad \alpha \equiv \operatorname{tr}\left(\llbracket C_{i} A_{j} \rrbracket\right)=\operatorname{tr}\left(\llbracket A_{i} C_{j} \rrbracket\right),
$$

$$
b \equiv \operatorname{tr}\left(\llbracket B_{j} \rrbracket\right)=\operatorname{tr}\left(\llbracket B_{i} \rrbracket\right), \quad \beta \equiv \operatorname{tr}\left(\llbracket C_{i} B_{j} \rrbracket\right)=\operatorname{tr}\left(\llbracket B_{i} C_{j} \rrbracket\right),
$$

$$
\gamma \equiv(1+a)(1+\beta)-\alpha b
$$

Equations (F1) and (F2) follow immediately from the properties $\llbracket A_{j} \rrbracket \cdot \llbracket A_{j} \rrbracket=a \llbracket A_{j} \rrbracket, \llbracket A_{i} \rrbracket \cdot \llbracket A_{i} \rrbracket=a \llbracket A_{i} \rrbracket, \llbracket B_{i} C_{j} \rrbracket \cdot$ $\llbracket B_{i} C_{j} \rrbracket=\beta \llbracket B_{i} C_{j} \rrbracket$, and $\llbracket C_{i} B_{j} \rrbracket \cdot \llbracket C_{i} B_{j} \rrbracket=\beta \llbracket C_{i} B_{j} \rrbracket$.

The proof of Eqs. (F3) and (F4) is somewhat more involved. We first multiply $\left(\mathrm{I}+\llbracket A_{j} \rrbracket\right)^{-1}$ by $\mathrm{I}+\llbracket A_{j} \rrbracket+\llbracket C_{i} B_{j} \rrbracket$ [see Eq. (F1)],

$$
\begin{aligned}
(\mathrm{I} & \left.+\llbracket A_{j} \rrbracket\right)^{-1} \cdot\left(\mathrm{I}+\llbracket A_{j} \rrbracket+\llbracket C_{i} B_{j} \rrbracket\right) \\
& =\mathrm{I}+\llbracket C_{i} B_{j} \rrbracket-\frac{\llbracket A_{j} \rrbracket \cdot \llbracket C_{i} B_{j} \rrbracket}{1+a}=\mathrm{I}+\llbracket C_{i}^{\prime} B_{j} \rrbracket,
\end{aligned}
$$

where, in the last step, we have used that $\llbracket A_{j} \rrbracket \cdot \llbracket C_{i} B_{j} \rrbracket=$ $\alpha \llbracket B_{j} \rrbracket$ and have introduced

$$
C_{i}^{\prime} \equiv C_{i}-\frac{\alpha}{1+a}
$$

Thus, the left inverse of $\mathrm{I}+\llbracket A_{j} \rrbracket+\llbracket C_{i} B_{j} \rrbracket$ is

$$
\begin{aligned}
(\mathrm{I} & \left.+\llbracket C_{i}^{\prime} B_{j} \rrbracket\right)^{-1} \cdot\left(\mathrm{I}+\llbracket A_{j} \rrbracket\right)^{-1} \\
& =\left(\mathrm{I}-\frac{\llbracket C_{i}^{\prime} B_{j} \rrbracket}{1+\beta^{\prime}}\right) \cdot\left(\mathrm{I}-\frac{\llbracket A_{j} \rrbracket}{1+a}\right),
\end{aligned}
$$

where

$$
\beta^{\prime} \equiv \operatorname{tr}\left(\llbracket C_{i}^{\prime} B_{j} \rrbracket\right)=\beta-\frac{\alpha b}{1+a},
$$

and use has been made of Eqs. (F1) and (F2a). Taking the property $\llbracket C_{i}^{\prime} B_{j} \rrbracket \cdot \llbracket A_{j} \rrbracket=b \llbracket C_{i}^{\prime} A_{j} \rrbracket$ into account, Eq. (F10) becomes

$$
\begin{aligned}
(\mathrm{I} & \left.+\llbracket C_{i}^{\prime} B_{j} \rrbracket\right)^{-1} \cdot\left(\mathrm{I}+\llbracket A_{j} \rrbracket\right)^{-1} \\
& =\mathrm{I}-\frac{\llbracket A_{j} \rrbracket}{1+a}-\frac{\llbracket C_{i}^{\prime} B_{j} \rrbracket}{1+\beta^{\prime}}+\frac{b}{\gamma} \llbracket C_{i}^{\prime} A_{j} \rrbracket,
\end{aligned}
$$

where we have used $\left(1+\beta^{\prime}\right)(1+a)=\gamma$. Finally, from Eq. (F9), it is straightforward to see that the right-hand side of Eqs. (F3) and (F12) coincide.

An analogous method can be used to prove that the righthand side of Eq. (F3) is also the right inverse of $\mathrm{I}+\llbracket A_{j} \rrbracket+$ $\llbracket C_{i} B_{j} \rrbracket$. Multiplying the latter matrix by $\left(I+\llbracket A_{j} \rrbracket\right)^{-1}$ [see Eq. (F1)], one gets

$$
\left(\mathrm{I}+\llbracket A_{j} \rrbracket+\llbracket C_{i} B_{j} \rrbracket\right) \cdot\left(\mathrm{I}+\llbracket A_{j} \rrbracket\right)^{-1}=\mathrm{I}+\llbracket C_{i} B_{j}^{\prime} \rrbracket,
$$
with

$$
B_{j}^{\prime} \equiv B_{j}-\frac{b}{1+a} A_{j} .
$$

Thus, the right inverse of $\mathrm{I}+\llbracket A_{j} \rrbracket+\llbracket C_{i} B_{j} \rrbracket$ is

$$
\begin{aligned}
(\mathrm{I} & \left.+\llbracket A_{j} \rrbracket\right)^{-1} \cdot\left(\mathrm{I}+\llbracket C_{i} B_{j}^{\prime} \rrbracket\right)^{-1} \\
& =\mathrm{I}-\frac{\llbracket A_{j} \rrbracket}{1+a}-\frac{\llbracket C_{i} B_{j}^{\prime} \rrbracket}{1+\beta^{\prime}}+\frac{\alpha}{\gamma} \llbracket B_{j}^{\prime} \rrbracket .
\end{aligned}
$$

Here, we have used $\beta^{\prime}=\operatorname{tr}\left(\llbracket C_{i} B_{j}^{\prime} \rrbracket\right.$ and $\llbracket A_{j} \rrbracket \cdot \llbracket C_{i} B_{j}^{\prime} \rrbracket=$ $\alpha \llbracket B_{j}^{\prime} \rrbracket$. Replacement of Eq. (F14) makes the right-hand side of Eq. (F15) coincide with the right-hand side of Eq. (F3). This completes the proof of Eq. (F3). Equation (F4) is just the transpose of Eq. (F3). Finally, note that Eq. (F3) reduces to Eqs. (F1) and (F2a) by particularizing to $C_{i}=0$ and $A_{i}=0$, respectively.
[1] Theory and Simulation of Hard-Sphere Fluids and Related Systems, edited by A. Mulero, Lectures Notes in Physics Vol. 753 (Springer, Berlin, 2008).

[2] F. H. Ree and W. G. Hoover, J. Chem. Phys. 40, 2048 (1964).

[3] B. C. Freasier and D. J. Isbister, Mol. Phys. 42, 927 (1981).

[4] M. Luban and A. Baram, J. Chem. Phys. 76, 3233 (1982).

[5] C. G. Joslin, J. Chem. Phys. 77, 2701 (1982).

[6] J. P. J. Michels and N. J. Trappeniers, Phys. Lett. A 104, 425 (1984).

[7] E. Leutheusser, Physica A 127, 667 (1984).

[8] M. Bishop, J. P. J. Michels, and I. M. de Schepper, Phys. Lett. A 111, 169 (1985).
[9] H. L. Frisch, N. Rivier, and D. Wyler, Phys. Rev. Lett. 54, 2061 (1985).

[10] H. L. Frisch, N. Rivier, and D. Wyler, Phys. Rev. Lett. 56, 2331 (1986); 57, 1192(E) (1986).

[11] E. Leutheusser, J. Chem. Phys. 84, 1050 (1986).

[12] W. Klein and H. L. Frisch, J. Chem. Phys. 84, 968 (1986).

[13] J. L. Colot and M. Baus, Phys. Lett. A 119, 135 (1986).

[14] H. L. Frisch and J. K. Percus, Phys. Rev. A 35, 4696 (1987).

[15] Y. Rosenfeld, J. Chem. Phys. 87, 4865 (1987).

[16] D. Wyler, N. Rivier, and H. L. Frisch, Phys. Rev. A 36, 2422 (1987).

[17] M. Baus and J. L. Colot, Phys. Rev. A 36, 3912 (1987).

[18] Y. Rosenfeld, J. Chem. Phys. 89, 4272 (1988). 
[19] B. Bagchi and S. A. Rice, J. Chem. Phys. 88, 1177 (1988).

[20] Y. Elskens and H. L. Frisch, Phys. Rev. A 37, 4351 (1988).

[21] Y. Song, E. A. Mason, and R. M. Stratt, J. Phys. Chem. 93, 6916 (1989).

[22] J. Amorós, J. R. Solana, and E. Villar, Phys. Chem. Liq. 19, 119 (1989).

[23] M. Luban and J. P. J. Michels, Phys. Rev. A 41, 6796 (1990).

[24] Y. Song and E. A. Mason, J. Chem. Phys. 93, 686 (1990).

[25] D. J. González, L. E. González, and M. Silbert, Phys. Chem. Liq. 22, 95 (1990).

[26] D. J. González, L. E. González, and M. Silbert, Mol. Phys. 74, 613 (1991).

[27] M. J. Maeso, J. R. Solana, J. Amorós, and E. Villar, Mater. Chem. Phys. 30, 39 (1991).

[28] J. G. Loeser, Z. Zhen, S. Kais, and D. R. Herschbach, J. Chem. Phys. 95, 4525 (1991).

[29] H.-O. Carmesin, H. L. Frisch, and J. K. Percus, J. Stat. Phys. 63, 791 (1991).

[30] L. E. González, D. J. González, and M. Silbert, J. Chem. Phys. 97, 5132 (1992).

[31] H. L. Frisch and J. K. Percus, Phys. Rev. E 60, 2942 (1999).

[32] E. Velasco, L. Mederos, and G. Navascués, Mol. Phys. 97, 1273 (1999).

[33] M. Bishop, A. Masters, and J. H. R. Clarke, J. Chem. Phys. 110, 11449 (1999).

[34] A. Santos, S. B. Yuste, and M. López de Haro, Mol. Phys. 96, 1 (1999).

[35] K. K. Mon and J. K. Percus, J. Chem. Phys. 110, 2734 (1999).

[36] G. Parisi and F. Slanina, Phys. Rev. E 62, 6554 (2000).

[37] S. B. Yuste, A. Santos, and M. López de Haro, Europhys. Lett. 52, 158 (2000).

[38] A. Santos, J. Chem. Phys. 112, 10680 (2000).

[39] A. Yukhimets, H. L. Frisch, and J. K. Percus, J. Stat. Phys. 100, 135 (2000).

[40] M. González-Melchor, J. Alejandre, and M. López de Haro, J. Chem. Phys. 114, 4905 (2001).

[41] R. Finken, M. Schmidt, and H. Löwen, Phys. Rev. E 65, 016108 (2001).

[42] A. Santos, S. B. Yuste, and M. López de Haro, Mol. Phys. 99, 1959 (2001).

[43] A. Santos, S. B. Yuste, and M. López de Haro, J. Chem. Phys. 117, 5785 (2002).

[44] E. Enciso, N. G. Almarza, M. A. Gonzalez, and F. J. Bermejo, Mol. Phys. 100, 1941 (2002).

[45] M. Robles, M. López de Haro, and A. Santos, J. Chem. Phys. 120, 9113 (2004).

[46] M. Bishop, A. Masters, and A. Y. Vlasov, J. Chem. Phys. 121, 6884 (2004).

[47] N. Clisby and B. M. McCoy, J. Stat. Phys. 114, 1343 (2004); 114, 1361 (2004).

[48] N. Clisby and B. M. McCoy, Pramana 64, 775 (2005).

[49] I. Lyberg, J. Stat. Phys. 119, 747 (2005).

[50] A. Santos and M. López de Haro, Phys. Rev. E 72, 010501(R) (2005).

[51] L. Lue, J. Chem. Phys. 122, 044513 (2005).

[52] M. Bishop, P. A. Whitlock, and D. Klein, J. Chem. Phys. 122, 074508 (2005).
[53] M. Bishop, A. Masters, and A. Y. Vlasov, J. Chem. Phys. 122, 154502 (2005).

[54] M. Bishop and P. A. Whitlock, J. Chem. Phys. 123, 014507 (2005).

[55] N. Clisby and B. M. McCoy, J. Stat. Phys. 122, 15 (2006).

[56] L. Lue and M. Bishop, Phys. Rev. E 74, 021201 (2006).

[57] M. Skoge, A. Donev, F. H. Stillinger, and S. Torquato, Phys. Rev. E 74, 041127 (2006).

[58] S. Torquato and F. H. Stillinger, Phys. Rev. E 73, 031106 (2006).

[59] S. Torquato and F. H. Stillinger, Exp. Math. 15, 307 (2006).

[60] S. Torquato, O. U. Uche, and F. H. Stillinger, Phys. Rev. E 74, 061308 (2006).

[61] G. Parisi and F. Zamponi, J. Stat. Mech. (2006) P03017.

[62] M. Robles, M. López de Haro, and A. Santos, J. Chem. Phys. 126, 016101 (2007).

[63] M. Bishop and P. A. Whitlock, J. Stat. Phys. 126, 299 (2007).

[64] P. A. Whitlock, M. Bishop, and J. L. Tiglias, J. Chem. Phys. 126, 224505 (2007).

[65] R. D. Rohrmann and A. Santos, Phys. Rev. E 76, 051202 (2007).

[66] M. Bishop, N. Clisby, and P. A. Whitlock, J. Chem. Phys. 128, 034506 (2008).

[67] M. Adda Bedia, E. Katzav, and D. Vella, J. Chem. Phys. 129, 144506 (2008).

[68] A. Scardicchio, F. H. Stillinger, and S. Torquato, J. Math. Phys. 49, 043301 (2008).

[69] R. D. Rohrmann, M. Robles, M. López de Haro, and A. Santos, J. Chem. Phys. 129, 014510 (2008).

[70] J. A. van Meel, D. Frenkel, and P. Charbonneau, Phys. Rev. E 79, 030201 (2009).

[71] J. A. van Meel, B. Charbonneau, A. Fortini, and P. Charbonneau, Phys. Rev. E 80, 061110 (2009).

[72] L. Lue, M. Bishop, and P. A. Whitlock, J. Chem. Phys. 132, 104509 (2010).

[73] S. Torquato and F. H. Stillinger, Rev. Mod. Phys. 82, 2633 (2010).

[74] C. D. Estrada and M. Robles, J. Chem. Phys. (in press).

[75] J. K. Percus and G. J. Yevick, Phys. Rev. 110, 1 (1958).

[76] F. Zernike and J. A. Prins, Z. Phys. 41, 184 (1927).

[77] M. S. Wertheim, Phys. Rev. Lett. 10, 321 (1963); J. Math. Phys. 5, 643 (1964).

[78] E. Thiele, J. Chem. Phys. 39, 474 (1963).

[79] M. Adda Bedia, E. Katzav, and D. Vella, J. Chem. Phys. 128, 184508 (2008).

[80] J. L. Lebowitz, Phys. Rev. 133, A895 (1964).

[81] S. B. Yuste, A. Santos, and M. López de Haro, J. Chem. Phys. 108, 3683 (1998).

[82] J. L. Lebowitz, J. K. Percus, and I. J. Zucker, Bull. Am. Phys. Soc. 7, 415 (1962).

[83] S. B. Yuste and A. Santos, Phys. Rev. A 43, 5418 (1991).

[84] M. López de Haro, S. B. Yuste, and A. Santos, in Theory and Simulation of Hard-Sphere Fluids and Related Systems, Alternative Approaches to the Equilibrium Properties of HardSphere Liquids, (Ref. [1]), p. 183.

[85] S. B. Yuste and A. Santos, J. Stat. Phys. 72, 703 (1993).

[86] S. B. Yuste and A. Santos, Phys. Rev. E 48, 4599 (1993).

[87] A. Santos, S. B. Yuste, and M. López de Haro, J. Chem. Phys. 109, 6814 (1998).

[88] S. B. Yuste and A. Santos, J. Chem. Phys. 101, 2355 (1994). 
[89] L. Acedo and A. Santos, J. Chem. Phys. 115, 2805 (2001).

[90] Al. Malijevský, S. B. Yuste, and A. Santos, Phys. Rev. E 76, 021504 (2007).

[91] N. W. Ashcroft and D. C. Langreth, Phys. Rev. 156, 685 (1967).
[92] Note that, if $\sigma_{i j}=1 \forall i, j$, then Eq. (5.21) yields the relation $a_{1}=$ $3\left[1-\eta\left(1+10 a_{2}\right)\right] /(1-6 \eta)\left(\mathrm{L}_{\ell}=a_{\ell} \llbracket 1 \rrbracket, \ell=1,2\right)$ earlier obtained for one-component fluids [65].

[93] A. B. Bathia and D. E. Thornton, Phys. Rev. B 2, 3004 (1970). 